\title{
Asymptotic behaviour of fundamental solutions of elliptic operators with order higher than two
}

\author{
Susumu TANABÉ \\ (Received August 18, 1996)
}

\begin{abstract}
One studies asymptotic behaviour at infinity of fundamental solutions to elliptic PDE order higher than two. The main tool to be used is estimates for oscillatory integrals obtained by A.N. Varchenko.
\end{abstract}

Key words: partial differential operator of elliptic type, singularity theory, asymptotic analysis.

\section{Introduction}

In this article, we shall investigate the following problem. Let $F(D)$ be a homogeneous elliptic differential operator of order $2 m(m \geq 2)$ with constant coefficients on $\mathbb{R}^{N}$ :

$$
F(D)=\sum_{|\alpha|=2 m} F_{\alpha} D^{\alpha}
$$

where $F(\xi)>0$ for $\xi \in \mathbb{R}^{N} \backslash 0, F_{\beta}=1$ for $\beta=(0,0, \ldots 0,2 \stackrel{i}{m}, 0, \ldots 0)$, $1 \leq i \leq N$.

Let $E(x)$ be the fundamental solution to the operator $F(D)-I$ that is bounded as $x$ tends to infinity,

$$
F(D) E(x)-E(x)=\delta(x) .
$$

The problem is to know asymptotic behaviour of $E(x)$ as $x$ tends to infinity, starting from the coefficients $F_{\alpha}, \alpha \in \mathbb{N}^{N}$. In the case when the energy surface $S=\{F(\xi)=1\}$ has no point where its total curvature vanishes, the answer to this problem has already been gotten by B.R. Vainberg [Vai], as we shall review in $\S 1$. Therefore we are interested in the cases where the total curvature may vanish at some points on the energy surface.

In $\S 1$ we remember the fundamental tools that help us to reduce the study on $E(x)$ to that of oscillatory integrals with degenerate phases.

1991 Mathematics Subject Classification : Primary 35J52; Secondary 35P32. 
In $\S 2$ we will treat general operators of the following form:

$$
\begin{aligned}
& F(\xi)=\xi_{1}^{2 m}+\sum_{\substack{|\alpha|+j=2 m,|\alpha|>0, j>0}} a_{j \alpha} \xi_{1}^{j} \xi^{\prime \alpha}+\xi_{2}^{2 m} \cdots+\xi_{N}^{2 m} \\
& \xi^{\prime}=\left(\xi_{2} \cdots \xi_{N}\right), m \geq 2, \\
& \alpha=\left(\alpha_{2}, \alpha_{3}, \ldots \alpha_{N}\right) \in \mathbb{N}^{N-1},
\end{aligned}
$$

where the coefficients $a_{j \alpha}$ will be assumed to be small enough so that the positivity of $F(\xi)$ is guaranteed everywhere except the origin and that $F\left(1, \xi^{\prime}\right)$ appears to be a strong Min-function (Definition 2.6) with some supplementary conditions (Condition 2.8). In this situation, we give an estimate on the decaying order of $E(x)$ as $x \rightarrow \infty$ with $|x /| x|-(1,0 \ldots 0)|<\varepsilon$ for some $\varepsilon>0$ (Theorem 2.11):

$$
|E(x)| \leq|x|^{-1 / d(\Gamma)}(\log |x|)^{\bar{\ell}(\Gamma)},
$$

where the meaning of notations $\Gamma, d(\Gamma)$ and $\bar{\ell}(\Gamma)$ will be explained in Proposition 2.3 and Theorem 2.5. The statement of Theorem 2.11 can be interpreted as an affirmation of the upper semicontinuity (with respect to $x$-variables) of the oscillatory index for oscillatory integrals of type

$$
\int \varphi\left(\xi^{\prime}\right) e^{i\left(x_{1} f\left(\xi^{\prime}\right)+x_{2} \xi_{2}+\cdots+x_{N} \xi_{N}\right)} d \xi^{\prime}
$$

where $f\left(\xi^{\prime}\right)$ is a strong Min-function on $\mathbb{R}^{N-1}$ with $d f(0)=0$. We shall remark here that it has been pointed out by Varchenko [Var, example 1], the upper semicontinuity of the oscillatory index does not hold for deformations of a smooth function $f\left(\xi^{\prime}\right)$ in general.

Moreover we give a result on the stratification structure of

$$
\left\{x \in \mathbb{R}^{N} ;|x /| x|-(1,0 \ldots 0)|<\varepsilon \text { for some } \varepsilon>0\right\},
$$

under the condition that the principal part of $f\left(\xi^{\prime}\right)$ consists only of terms with even powers and positive coefficients. Under this condition, on each stratum

$$
\begin{aligned}
\sigma_{I}=\left\{x \in \mathbb{R}^{N} ; x_{i} \neq 0 \text { for } i \in I \text { and } x_{j}=0 \text { for } j \notin I,\right. \\
\text { where } 1 \in I \subset\{1, \ldots, N\}\},
\end{aligned}
$$

of this stratification we have

$$
|E(x)| \leq C(\omega)|x|^{(I-1) / 2-1 / d(\Gamma(I))}(\log |x|)^{\bar{\ell}(\Gamma(I))},
$$


(Theorem 2.11).

In $\S 3$ we treat 4 -th order elliptic operators on $\mathbb{R}^{2}$ i.e.

$$
F(\xi)=\xi_{1}^{4}+p \xi_{1}^{2} \xi_{2}^{2}+q \xi_{1} \xi_{2}^{3}+r \xi_{2}^{4} .
$$

Here we impose no condition on the coefficients $(p, q, r)$ except the one which guarantees the positivity of $(0.3)$. In contrast with $\S 1$, no restriction on the size of the coefficients $(p, q, r)$ is assumed.

Evidently when $p=q=0$ and $r>0$, the curvature of surface (curve in this case) $S$ vanishes at the points $\left(\xi_{1}, \xi_{2}\right)=( \pm 1,0)$ and $\left(0, \pm r^{-1 / 4}\right)$. But in general cases where the coefficients $p$ and $q$ are different from zero, it is not so easy to see what kind of vanishing of the curvature may take place on $S$. Therefore at first we give a classification of the quadratic curves in terms of the coefficients $(p, q, r)$ in Proposition 3.5, then we obtain the asymptotic behaviour of the oscillatory integral whence that of $E(x)$ can be obtained (Theorem 3.7).

In $\S 4$, we apply the results of $\S 3$ to estimates of 1 ) error term in Weyl type formula and 2) the eigenfunctions of Dirichlet problem to an elliptic operator $F(D)$ like $(0.3)$.

We would like to mention here that the problem 2) above was the basic motivation of the investigations of this paper. This setting of question was given by Yu.V. Egorov during my stay at the Moscow State University in 1990. The author expresses his hearty gratitude to Prof. Akira Kaneko, and to the referee for detailed proof reading.

\section{Preparatory propositions}

First of all, we shall prove and review results about the energy surface $S$ so as to use them in subsequent situations.

Lemma 1.1 $F(\xi)$ is a strictly positive function as in (0.1), the energy surface $S=\left\{\xi \in \mathbb{R}^{N} ; F(\xi)=1\right\}$ is a connected and analytic hypersurface in $\mathbb{R}^{N}$. Furthermore the inhomogeneous function $F(\xi)-1$ has the following factorization in some neighbourhood of every point $\stackrel{\circ}{\xi} \in S \backslash\left\{\xi_{i}=0\right\}$ :

$$
F(\xi)-1=G_{i}(\xi) T_{i}(\xi)
$$

where $G_{i}(\xi)$ is analytic function of the form $G_{i}(\xi)=\xi_{i}^{2}-\widetilde{G}_{i}\left(\xi^{\prime}\right)$, and it vanishes on $S, \xi^{\prime}=\left(\xi_{1}, \ldots, \check{\xi}_{i}, \ldots, \xi_{N}\right),\left|\nabla G_{i}(\xi)\right| \neq 0$ on $S$ and $T_{i}(\xi) \neq 0$ on $S$. 
Proof. Let us assume that $\min _{|\xi|=1} F(\xi)=F(\stackrel{\circ}{\xi})=c>0$. Because of the positivity and homogeneity of $F$, we have $F(\xi)=|\xi|^{2 m} F(\xi /|\xi|)>c|\xi|^{2 m}$ for all $\xi$. This means the boundedness of the surface $S$. Furthermore for each direction $\omega \in S^{N-1}$ one can always find unique value $\widetilde{t}$ such that $F(\widetilde{t} \omega)=2 c$. This implies the connectedness of the surface $S$.

As for factorization (1.1), we may choose the analytic function $\widetilde{G}_{i}\left(\xi^{\prime}\right)$ as follows. As it is easily seen from the non-vanishing property of $\nabla F(\xi)$ on $S$, for every $\stackrel{\circ}{\xi} \in S$ one can choose an index $i$ such that $\frac{\partial}{\partial \xi_{i}} F(\xi) \neq 0$. This implies that $S$ can be considered as a graph of a function $\xi_{i}\left(\xi^{\prime}\right), \xi_{i}=\xi_{i}\left(\xi^{\prime}\right)$, in some neighbourhood of the point $\stackrel{\circ}{\xi}$ where $\frac{\partial}{\partial \xi_{i}} F(\xi)$ does not vanish. Further we suppose $i=1$ by juxtaposition of the indices of variables. While the roots of equation $F\left(\xi_{1}, \xi^{\prime}\right)=1$ depends on $\xi^{\prime}$ continuously, the function $\xi_{1}\left(\xi^{\prime}\right)$ that describes the surface $S$ in the neighbourhood of the point $\stackrel{\circ}{\xi}$ can be expressed by the following complex integral:

$$
\xi_{1}\left(\xi^{\prime}\right)=\frac{1}{2 \pi i} \int_{\gamma} \frac{z F_{z}\left(z, \xi^{\prime}\right)}{F\left(z, \xi^{\prime}\right)-1} d z
$$

where $\gamma$ denotes a circle on $z$ complex plane with center $z=\stackrel{\circ}{\xi}_{1}$ that does not touch the other roots of $F(z, \stackrel{\circ}{\xi})=1$. We may express the function $\xi_{1}\left(\xi^{\prime}\right)$ in terms of the above integral by moving the circle $\gamma$ towards left side and shrinking it not to touch the other roots of the equation with respect to $z, F(z, \stackrel{\circ}{\xi})=1$. Thus we have shown that the surface $\left.S\right|_{\xi_{1}<0}$ can be expressed by an analytic function $\xi_{1}=\xi_{1}\left(\xi^{\prime}\right)$ in some neighbourhood of the point $\stackrel{\circ}{\xi}$. The other half of the surface $\left.S\right|_{\xi_{1}>0}$ also has a similar expression $\widetilde{\xi}_{1}\left(\xi^{\prime}\right)=-\xi_{1}\left(-\xi^{\prime}\right)$ in the neighbourhood of the antipodal point $\stackrel{\circ}{\xi}$, and we have only to set $\widetilde{G}_{1}\left(\xi^{\prime}\right)=\xi_{1}\left(\xi^{\prime}\right) \widetilde{\xi}_{1}\left(\xi^{\prime}\right)$. The non-vanishing property of $\nabla G(\xi)$ on $S$ can be shown as follows. It is easy to see that

$$
\nabla G(\xi)=\frac{\nabla F(\xi)}{T(\xi)} \quad \text { on } \quad S
$$

by differentiating the expression $F=G \cdot T$. From the Euler equality

$$
\sum_{j=1}^{N} \xi_{j} \frac{\partial F}{\partial \xi_{j}}(\xi)=2 m F(\xi)
$$


the non-nullity of $\nabla F$ can be deduced. Hence $\nabla G(\xi) \neq 0$ on $S$.

Let us assume that the direction $\omega=x /|x|$ is parallel to the normal to the surface $S$ at a point $\xi^{*}$. We may suppose that a function $G(\xi)$ as Lemma 1.1 describes the surface $S$ in some neighbourhood of the surface $S$ in $\mathbb{R}_{\xi}^{N}$ swept by the surface $S(t),-\delta \leq t \leq \delta$ where $S(t)=\{F(\xi)=1+t\}$. Let $\varphi(\xi) \in \mathcal{C}_{0}^{\infty}\left(\mathbb{R}^{N}\right)$ be a function that vanishes outside $U$ and $\varphi(\xi)=1$ for $\xi \in U$ with $|F(\xi)-1|<\delta / 2$. According to [Vai], there exists a fundamental solution $E(x)$ that can be expressed as follows:

$$
\begin{aligned}
E(x)=(2 \pi)^{-N} & \left(\text { v.p. } \int_{U} \frac{\varphi(\xi)}{F(\xi)-1} e^{-i x \xi} d \xi\right. \\
& \left.-\pi i \int_{S} \frac{e^{-i x \sigma}}{T(\sigma)|\nabla F(\sigma)|} d S\right)+R(x)
\end{aligned}
$$

where $R(x)$ denotes a rapidly decreasing smooth function. In this way to get an asymptotic behaviour of the fundamental solution $E(x)$, we are asked to know that of the above two integral expressions. For this, we write the integral over $U$ as a repeated integral, first over the surface $S(t)$ and then with respect to $t$. If we denote the line element of the normal to $S(t)$ by $d \ell$, then $d \ell=d T /|\nabla F(\sigma)|$. Therefore the volume element $d \xi=\frac{d S d t}{|\nabla F|}$, where $d S$ denotes the surface element of $S$. We set

$$
\Phi(t, \omega, r)=-i(2 \pi)^{1-N} \int_{S(t)} \frac{\varphi(\xi) e^{-i x \xi}}{T(\xi)|\nabla F(\xi)|} d S
$$

where $\omega=x /|x|, r=|x|$. Then the fundamental solution can be written down as follows:

$$
E(x)=\frac{1}{2}\left(\frac{-1}{\pi i} \mathrm{v} \cdot \mathrm{p} \cdot \int_{-\delta}^{\delta} \frac{\Phi(t, \omega, r)}{t} d t+\Phi(0, \omega, r)\right)+R(x) .
$$

Before going into further analysis of these integrals, we recall a lemma from [Vai]

Lemma 1.2 Suppose that $\phi(\omega, t), f(\omega, t) \in \mathcal{C}^{\infty}\left(S^{N-1} \times \mathbb{R}^{1}\right), \phi$ is real valued, $f=0$ for $t>\delta>0$ and that $\phi_{t}^{\prime} \neq 0$ for $|t| \leq \delta$. Then

v.p. $\int t^{-1} f(\omega, t) e^{i r \phi(\omega, t)} d t=\left[\operatorname{sign} \phi_{t}^{\prime}(\omega, 0)\right] i \pi f(\omega, 0) e^{i \phi(\omega, 0) r}+F(\omega, r)$

Here $F(\omega, r)$ satisfies the following estimate: For every $(\alpha, j) \in \mathbb{N}^{N} \times \mathbb{N}^{1}$, 
there exists a positive constant $C_{\alpha, j}$ such that

$$
\left|\left(\frac{\partial}{\partial \omega}\right)^{\alpha}\left(\frac{\partial}{\partial r}\right)^{j} F(\omega, r)\right| \leq C_{\alpha, j} r^{-L} \quad \text { for any } L>0 .
$$

By the aid of the above lemma, we have only to know asymptotic behaviour of $\Phi(0, \omega, r)$ in order to get that of $E(x)$. Thus the problem is reduced to determine the asymptotic behaviour of the integral of type

$$
I(x)=\int_{S} f(\sigma) e^{-i x \sigma} d S .
$$

In the case that the curvature of the surface $S$ never vanishes, the following result is known.

Theorem 1.3 [Vai] Suppose that on the surface $S$ there is a finite number of points $\sigma^{j}\left(\omega_{0}\right),(j=1, \ldots, J)$ at which the normal vector to the surface $S$ is parallel to the vector $\omega_{0}$. Assume that at the point $\sigma\left(\omega_{0}\right)$ the curvature of $S$ does not vanish. In this situation there exists $\varepsilon>0$ such that for $\left|\omega-\omega_{0}\right|<0$, the following asymptotic expansion holds modulo rapidly decreasing smooth functions.

$$
\Phi(0, \omega, r) \sim \sum_{j=1}^{J} e^{-i \mu_{j}(\omega) r}\left\{\sum_{s=0}^{\infty} a_{j s} r^{(1-r) / 2-s}\right\}
$$

where

$$
a_{j s}=(2 \pi)^{(N-1) / 2}\left|\kappa_{j}(\omega)\right|^{-1 / 2} e^{-\gamma_{j} \pi / 4} .
$$

$\kappa_{j}$ : the curvature of $S$ at the point $\sigma^{j}(\omega)$.

$\mu_{j}(\omega)=\left\langle\sigma^{j}, \omega\right\rangle$ : the projection of the point $\sigma^{j}(\omega)$ on the line parallel to $\omega$ passing through the origin.

$\gamma_{j}$ : inertia index of the curvature form at $\sigma^{j}(\omega)$.

We review the proof of this theorem so as to use it in our problem. Let $\{\phi\}$ be the partition of the unity on $S$ subordinate to covering $U_{j}$. We choose $U_{j}$ in such a way that on every $U_{j}$ there is only one point $\sigma^{j}(\omega)$ at which the normal to the surface $S$ is parallel to $\omega$.

We adopt such a coordinate system $\widetilde{\xi}$ that $S$ can be written down in the form $\widetilde{\xi}_{1}=\widetilde{\xi}_{1}\left(\widetilde{\xi}^{\prime}\right)$ on $U_{j}$, where $\widetilde{\xi}^{\prime}=\left(\widetilde{\xi}_{2}, \ldots, \widetilde{\xi}_{N}\right) \in V_{j} \subset \mathbb{R}^{N-1}$. $\sigma(\widetilde{\xi})=\left(\widetilde{\xi}_{1}\left(\widetilde{\xi}^{\prime}\right), \widetilde{\xi}_{2}^{\prime}, \ldots, \widetilde{\xi}_{N}^{\prime}\right)$. 
Then we have:

$$
\begin{aligned}
\Phi(0, \omega, r) & =\sum_{j=1}^{J} \int_{U_{j}} \phi(\sigma) f(\sigma) e^{-i r \omega \sigma} d S \\
& =\sum_{j=1}^{J} \int_{V_{j}} \phi(\sigma(\widetilde{\xi})) f(\sigma(\widetilde{\xi})) e^{-i r \omega \sigma(\widetilde{\xi})} d \widetilde{\xi} .
\end{aligned}
$$

Thus the phase function $r \omega \sigma(\widetilde{\xi})$ reads as $r \widetilde{\xi}_{1}\left(\widetilde{\xi}^{\prime}\right)$ in this coordinate system. Hence the problem has been reduced to know the asymptotic behaviour of the oscillatory integrals like (1.6) with $\omega \sigma(\widetilde{\xi})=\widetilde{\xi}_{1}\left(\widetilde{\xi}^{\prime}\right)$. As is easily seen, under the non-vanishing assumption on the curvature of $S$ such kind of integrals can be calculated with use of ordinary version of the stationary phase method with nondegenerate phases.

In the following sections, the information of the Taylor's expansion of the function $\xi_{1}\left(\widetilde{\xi}^{\prime}\right)$ with respect to $\widetilde{\xi}^{\prime}$ will be studied as our main subject.

\section{Local estimates of the fundamental solution of the elliptic operator in general case}

Let us consider a symbol of homogeneous elliptic operator of order $2 m$ :

$$
F_{a}(\xi)=F_{0}(\xi)+\sum_{\substack{|\alpha|+j=2 m \\ 0<j<2 m}} a_{j \alpha} \xi_{1}^{j} \xi^{\alpha}
$$

where

$$
F_{0}(\xi)=\xi_{1}^{2 m}+\xi_{2}^{2 m}+\cdots+\xi_{N}^{2 m}, \quad m \geq 2 .
$$

and $\sum_{|\alpha|+j=2 m}\left|a_{j \alpha}\right|<1$. We have imposed this condition so that the positivity of $F$ except the origin should hold.

In this section, we shall study the asymptotic behaviour of the fundamental solution $E(x)$ of the operator $F_{a}(D)-I$, that is bounded as $x$ tends to infinity, i.e.

$$
\left(F_{a}(D)-I\right) E(x)=\delta(x)
$$

Let us regard $F_{a}(\xi)$ a a function depending on $N$ dimensional variables $\xi$ and $L$ dimensional variables $a$, where $L={ }_{N+2 m-1} C_{N}$. Then we may regard the germ $\xi_{1}\left(\xi^{\prime}, a\right)$, representing the branch of the root of the equation $F_{a}\left(\xi_{1}, \xi^{\prime}\right)=1$ with $\xi_{1}(0,0)=1$, as a real valued continuous function on 


$$
D=\left\{\left(\xi^{\prime}, a\right) \in \mathbb{R}^{N} \times \mathbb{R}^{L} ;\left|\xi^{\prime}\right|<\delta,|a|<\delta\right\} .
$$

Lemma 2.1 The function germ $\xi_{1}\left(\xi^{\prime}, a\right)$ satisfying $\xi_{1}(0,0)=1$ is an analytic germ on $D$.

One can prove this lemma by means of a similar argument to that of Lemma 1.1, in using an integral expression of the germ $\xi_{1}\left(\xi^{\prime}, a\right)$ :

$$
\xi_{1}\left(\xi^{\prime}, a\right)=\frac{1}{2 \pi i} \int_{\gamma} \frac{z \frac{\partial}{\partial z} F_{a}\left(z, \xi^{\prime}\right)}{F_{a}\left(z, \xi^{\prime}\right)-1} d z
$$

To state further information on $\xi_{1}\left(\xi^{\prime}, a\right)$, we prepare the following fundamental notions on analytic functions.

Definition 2.2 (Newton's diagram) Let $f(\xi)=\sum_{\alpha \in \mathbb{N}^{N}} a_{\alpha} \xi^{\alpha}$ be an analytic function in some neighbourhood of the origin.

We temporarily use the notation $\operatorname{supp} f=\left\{\alpha \in \mathbb{N}^{N} ; a_{\alpha} \neq 0\right\}$. Newton's polyhedron $\Gamma_{+}(f)$ of a series $f$ is defined by the convex hull in $\mathbb{R}_{+}^{N}$ of the set $\bigcup_{\alpha \in \text { supp f }}\left(\alpha+\mathbb{R}_{+}^{N}\right)$. The union of all compact faces, of Newton's polyhedron $\Gamma_{+}(f)$ will be called Newton's diagram and denoted by $\Gamma(f)$. The principal part of a series $f$ is defined by the polynomial

$$
\widehat{f}=\sum_{\alpha \in \Gamma(f)} a_{\alpha} \xi^{\alpha} .
$$

For any compact face $\gamma \subset \Gamma(f)$ we will denote by $f_{\gamma}$ the polynomial

$$
f_{\gamma}=\sum_{\alpha \in \gamma} a_{\alpha} \xi^{\alpha}
$$

In order to know the informations on Newton's diagram and the principal part of the function $\xi_{1}\left(\xi^{\prime}, a\right)$, we employ a heuristic argument. Let us differentiate the equation $F_{a}(\xi)-1=0$ with respect to $a_{j \alpha}$ and we get,

$$
2 m \frac{\partial \xi_{1}}{\partial a_{j \alpha}}\left(\xi^{\prime}, a\right) \xi_{1}^{2 m-1}+\xi_{1}^{j} \xi^{\prime \alpha}+\sum_{|\beta|+k=2 m} k a_{k \beta} \frac{\partial \xi_{1}}{\partial a_{j \alpha}}\left(\xi^{\prime}, a\right) \xi_{1}^{k-1} \xi^{\prime \beta}=0
$$

or

$$
\begin{aligned}
& \frac{\partial \xi_{1}}{\partial a_{j \alpha}}\left(\xi^{\prime} \cdot a\right) \\
& \quad=-\xi_{1}^{j} \xi^{\prime \alpha} /\left\{2 m \xi_{1}^{2 m-1}+\sum_{|\beta|+k=2 m} k a_{k \beta} \frac{\partial \xi_{1}}{\partial a_{j \alpha}}\left(\xi^{\prime}, a\right) \xi_{1}^{k-1} \xi^{\prime \beta}\right\}
\end{aligned}
$$


We shall derive some informations on Taylor series of $\xi_{1}\left(\xi^{\prime}, a\right)$ with respect to $\left(\xi^{\prime}, a\right) \in \mathbb{R}^{N-1} \times \mathbb{R}^{L}$, from the above nonlinear equations.

As we consider the behaviour of $\xi_{1}\left(\xi^{\prime}, a\right)$ in the neighbourhood of $\left(\xi_{1}, \xi^{\prime}, a\right)=(1,0,0)$, we assume that $\xi_{1}\left(\xi^{\prime}, a\right)$ has a Taylor expansion

$$
\xi_{1}\left(\xi^{\prime}, a\right)=1+\sum_{1 \leq|\gamma|} \xi_{\gamma}(a) \xi^{\prime \gamma}
$$

in the neighbourhood of the origin after Lemma 2.1.

Therefore we have

$$
\begin{aligned}
\frac{\partial \xi_{1}}{\partial a_{j \alpha}}\left(\xi^{\prime}, a\right) & =-\xi_{1}^{j}{\xi^{\prime}}^{\alpha} /\left\{2 m+(2 m-1) \sum \xi_{\gamma}(a) \xi^{\prime \gamma}+\cdots\right\} \\
& =-\xi_{1}^{j}{\xi^{\prime}}^{\alpha} /\left\{2 m+\sum_{|\beta-\alpha|>0} b_{\beta} \xi^{\prime \beta}\right\} .
\end{aligned}
$$

These equalities hold in the sense of Taylor expansion for analytic function germs in the neighbourhood of $\left(\xi^{\prime}, a\right)=(0,0)$. Furthermore, to know the dependence of $\xi_{1}\left(\xi^{\prime}, a\right)$ on the variables $a$, we differentiate once more the above expression (2.2) with respect to $a_{i \mu}$. We get,

$$
\begin{aligned}
& \frac{\partial}{\partial a_{i \mu}} \cdot \frac{\partial}{\partial a_{j \alpha}} \xi_{1}\left(\xi^{\prime}, a\right) \\
& =-j \frac{\partial \xi_{1}}{\partial a_{i \mu}}\left(\xi^{\prime}, a\right) \cdot \xi_{1}^{j-1} \xi^{\prime \alpha} \\
& \quad \times\left\{2 m \xi_{1}^{2 m-1}+\sum_{|\beta|+k=2 m} k a_{k \beta} \frac{\partial \xi_{1}}{\partial a_{j \alpha}}\left(\xi^{\prime}, a\right) \xi_{1}^{k-1} \xi^{\prime \beta}\right\}^{-1} \\
& \quad-\xi_{1}^{j} \xi^{\prime \alpha}\left\{2 m(2 m-1) \xi_{1}^{2 m-2} \frac{\partial \xi_{1}}{\partial a_{i \mu}}\left(\xi^{\prime}, a\right)\right. \\
& \left.\quad+\frac{\partial \xi_{1}}{\partial a_{i \mu}} \sum_{|\beta|+k=2 m} k(k-1) a_{k \beta} \frac{\partial \xi_{1}}{\partial a_{j \alpha}}\left(\xi^{\prime}, a\right) \xi_{1}^{k-2} \xi^{\prime \beta}\right\} \\
& \left.=-j \frac{\partial \xi_{1}}{\partial a_{i \mu}}\left(\xi^{\prime}, a\right) \cdot \xi_{1}^{j-1} \xi^{\prime \alpha} \xi_{1}^{2 m-1}+\sum_{|\beta|+k=2 m} k a_{k \beta} \frac{\partial \xi_{1}}{\partial a_{j \alpha}}\left(\xi^{\prime}, a\right) \xi_{1}^{k-1} \xi^{\prime \beta}\right\}^{-2}+\cdots \\
& \quad \cdot\left[\left\{2 m \xi_{1}^{2 m-1}+\sum_{|\beta|+k=2 m} k a_{k \beta} \frac{\partial \xi_{1}}{\partial a_{j \alpha}}\left(\xi^{\prime}, a\right) \xi_{1}^{k-1} \xi^{\prime \beta}\right\}^{-1}\right.
\end{aligned}
$$




$$
\begin{aligned}
& -\frac{1}{j} \xi_{1}\left\{2 m(2 m-1) \xi_{1}^{2 m-2}\right. \\
& \left.+\sum_{|\beta|+k=2 m} k(k-1) a_{k \beta} \frac{\partial \xi_{1}}{\partial a_{j \alpha}}\left(\xi^{\prime}, a\right) \xi_{1}^{k-2} \xi^{\prime \beta}\right\} \\
& \left.\times\left\{2 m \xi_{1}^{2 m-1}+\sum_{|\beta|+k=2 m} k a_{k \beta} \frac{\partial \xi_{1}}{\partial a_{j \alpha}}\left(\xi^{\prime}, a\right) \xi_{1}^{k-1} \xi^{\prime \beta}\right\}^{-2}+\cdots\right] \\
& =\xi_{1}^{j-1} \xi^{\prime \alpha} \xi_{1}^{i} \xi^{\prime \mu} \cdot\left\{2 m \xi_{1}^{2 m-1}+\sum_{|\beta|+k=2 m} k a_{k \beta} \frac{\partial \xi_{1}}{\partial a_{j \alpha}}\left(\xi^{\prime}, a\right) \xi_{1}^{k-1} \xi^{\prime \beta}\right\}^{-1} \\
& \times\left[-j+\xi_{1}\left\{2 m(2 m-1) \xi_{1}^{2 m-2} k \sum_{|\beta|+k=2 m} k(k-1) a_{k \beta} \frac{\partial \xi_{1}}{\partial a_{j \alpha}}\left(\xi^{\prime}, a\right) \xi_{1}^{k-2} \xi^{\prime \beta}\right\}\right. \\
& \left.\times\left\{2 m \xi_{1}^{2 m-1}+\sum_{|\beta|+k=2 m} k a_{k \beta} \frac{\partial \xi_{1}}{\partial a_{j \alpha}}\left(\xi^{\prime}, a\right) \xi_{1}^{k-1} \xi^{\prime \beta}\right\}^{-1}+\cdots\right]
\end{aligned}
$$

Thus it is seen that if there is some term $\xi^{\prime \gamma}$ with coefficient including $a_{j \alpha} \cdot a_{j \mu}$, then $\gamma_{\ell}-\alpha_{\ell}-\mu_{\ell}>0$ for $2 \leq \ell \leq N$. Let us denote this kind of situation for the multi-indices by $[\gamma-\alpha-\mu]>0$. By the induction argument, for a term $a_{j_{1} \alpha_{1}} a_{j_{2} \alpha_{2}} \cdots a_{j_{p} \alpha_{p}} \xi^{\gamma}$ appearing in the Taylor expansion of $\xi_{1}\left(\xi^{\prime}, a\right)$, the equality $\left[\gamma-\alpha_{1}-\alpha_{2}-\cdots-\alpha_{p}\right]>0$ holds. Thus we have gotten the following:

Proposition 2.3 The function germ $\xi_{1}\left(\xi^{\prime}, a\right)$ with $\xi_{1}(0,0)=1$ has the following Taylor expansion in some small neighbourhood of the origin.

$$
\xi_{1}\left(\xi^{\prime}, a\right)=1-\frac{1}{2 m}\left\{\xi_{2}^{2 m}+\cdots+\xi_{N}^{2 m}+\sum_{\alpha \in \Gamma(f)} a_{j \alpha} \xi^{\prime \alpha}\right\}+R\left(\xi^{\prime}\right)
$$

where the Newton polyhedron of $R(\xi)$ is strictly contained in the set

$\Gamma_{+}=$convex hull of ( $\left\{\right.$the Newton polyhedron of $\left.f\left(\xi^{\prime}\right)=F\left(1, \xi^{\prime}\right)-1\right\}$

$$
\left.\bigcup\left\{\left(\gamma_{2}, \ldots, \gamma_{N}\right) ;\left|\gamma_{2}+\cdots \gamma_{N}\right|>2 m\right\}\right)
$$

To state the main theorem, we introduce some conditions that will be imposed on $F\left(1, \xi^{\prime}\right)$. 
In the sequel a real function germ $\varphi$ at the origin will always be assumed to satisfy $\varphi(0)=0$ and $d \varphi(0)=0$. Then, we will be interested in the following type of oscillatory integrals with phase function $\varphi(\xi)$ and an amplitude function $\chi(\xi)$ that has a very small compact support in the neighbourhood of the origin:

$$
I(\tau)=\int_{\mathbb{R}^{N}} e^{i \tau \varphi(\xi)} \chi(\xi) d \xi .
$$

As it is well known an oscillatory integral of type (2.5) has an asymptotic expansion in $\tau$ (see for example $[\mathrm{M}]$ ):

$$
I(\tau)=e^{i \tau \varphi(0)} \sum_{p} \sum_{k=1}^{N-1} a_{p k} \tau^{p}(\log \tau)^{k}
$$

where $p$ runs through finitely many arithmetic progressions independent of $\chi$.

We define the oscillation index of the asymptotic expansion (2.6) to be the number $\beta(f)$ that is the maximal of the numbers $p$ with following property:

For any neighbourhood of zero in $\mathbb{R}^{N}$, there exists a $\chi \in \mathcal{C}_{0}^{\infty}$ with support in this neighbourhood such that there exists an integer $k$ with $a_{p k} \neq 0$. Let us specify a coordinate system $\left(\xi_{1}, \ldots, \xi_{N}\right)$ on $\mathbb{R}^{N}$ and by $\varphi(\xi)$ the Taylor series of the function $\varphi$ at zero in this coordinate system. Let us denote by $d(\Gamma(\varphi))$ the values of the parameter of the intersection of the straight line $\alpha_{1}=\alpha_{2}=\cdots=\alpha_{N}=t, t \in \mathbb{R}_{+}$, with the boundary of the Newton polyhedron $\Gamma(\varphi)$. We call this number $d(\Gamma(\varphi))$ the height of the Newton polyhedron $\Gamma(\varphi)$.

Definition 2.4 The principal part of a series $\varphi(\xi)$ at $0 \in \mathbb{R}^{N}$ is said to be non-degenerate if for any closed face $\gamma \subset \Gamma(\varphi)$ the polynomials $\xi_{1} \frac{\partial \varphi_{\gamma}}{\partial \xi_{1}}, \ldots, \xi_{N} \frac{\partial \varphi_{\gamma}}{\partial \xi_{N}}$ do not vanish simultaneously on $\left\{\xi \in \mathbb{R}^{N} ; \xi_{1} \xi_{2} \cdots \xi_{N} \neq\right.$ $0\}$.

We shall review here the fundamental theorem for the asymptotic behaviour under question.

Theorem 2.5 ([Var]) Suppose that the principal part of the series $\varphi$ is non-degenerate at $\xi=0$.

Then

(1) There exist finitely many arithmetic progressions consisting of negative 
rational numbers which include all $p$ such that $a_{p k} \neq 0$.

(2) If the height of the Newton polyhedron is not larger than 1, then the oscillation index $\beta(\varphi)$ of (2.6) will not exceed $-1 / d \Gamma(\varphi)$.

(3) If the height of the Newton polyhedron is strictly larger than 1, then the oscillation index $\beta(\varphi)$ of (2.6) will be equal to $-1 / d \Gamma(\varphi)$.

(4) If the height of the Newton polyhedron is strictly larger than 1 and the point $(d(\Gamma), \ldots, d(\Gamma))$ lies at the intersection of $\ell$ pairs of $(N-1)$ dimensional faces of the Newton diagram $\Gamma(\widehat{\varphi})$, then for any nonnegative $\chi \in \mathcal{C}_{0}^{\infty}\left(\mathbb{R}^{N}\right)$ with $\chi(0) \neq 0$ and a support lying in a sufficiently small neighbourhood of zero in $\mathbb{R}^{n}$, we shall have a coefficient $a_{\beta(\varphi), \bar{\ell}-1}(\chi) \neq 0$ in (2.6), where $\bar{\ell}=\min \{\ell, N\}$. Moreover, in this situation we have $a_{\beta(\varphi), k}(\chi)=0$ for all $k \geq \bar{\ell}$.

As we shall treat the oscillatory integral like (1.6), we will introduce a class of function that may appear as the function germ $\xi_{1}\left(\xi^{\prime}, a\right)$ that we have investigated in the proof of Proposition 2.3.

Definition 2.6 An analytic function germ $\varphi(\xi) \in \mathcal{A}\left(\mathbb{R}^{N}, 0\right)$ at the origin that satisfies the following conditions will be called a strong Min-function.

(1) The Newton diagram $\Gamma(\varphi)$ meets all the coordinate axes and all of its vertices have only even coordinates.

(2) All the coefficients of the germ $\varphi$ corresponding to the vertices of $\Gamma(\varphi)$ are positive.

(3) For any face $\gamma \subset \Gamma(\varphi)$ of arbitrary dimension $\varphi_{\gamma}(\xi)>0$ for $\xi \neq 0$.

Remark 2.7 V.A. Vasiliev [Vas2] used the term of "Min-function" to denote the analytic function germ that has isolated minimum at the origin. Therefore all the strong Min-function defined above belong to the set of Min-function in the sense of Vasiliev.

From now on, let us impose the following condition on $F\left(1, \xi^{\prime}\right)$.

\section{Condition 2.8}

$$
f\left(\xi^{\prime}\right):=F\left(1, \xi^{\prime}\right)-1=\sum_{\substack{|\alpha|+j=2 m \\|\alpha|>0, j>0}} a_{j \alpha} \xi^{\prime \alpha}+\xi_{2}^{2 m}+\cdots+\xi_{N}^{2 m}
$$

is a strong Min-function of $(N-1)$-variables, and its Newton diagram contains no point of the form $(0, \ldots, \stackrel{i}{2}, \ldots, 0), 2 \leq i \leq N$. 
Under this assumption we see that the function germ $-\xi_{1}\left(1, \xi^{\prime}\right)$ also turns out to be a strong Min-function owing to Proposition 2.3.

Lemma 2.9 The principal part of a strong Min-function $\varphi$ is nondegenerate.

Proof. Take one of the faces $\gamma \subset \Gamma(\varphi)$. Assume that $\gamma$ lies on the coordinate plane $\left\{\alpha_{j_{J+1}}=\cdots=\alpha_{j_{N}}=0\right\}$. Without loss of generality we may suppose that $j_{J+1}=J+1, \ldots, j_{N}=N$. That is to say, there exists a point $\left(\alpha_{1}, \ldots, \alpha_{J}, 0, \ldots, 0\right) \in \gamma$ such that $a_{\alpha_{1} \cdots \alpha_{J}} \neq 0$. Let us show that $\varphi_{\gamma}$ cannot have singular points other than 0 . As is easily seen there exists integer $\ell(\gamma)$ such that

$$
\widehat{\varphi}_{\gamma}\left(\xi_{1}, \ldots, \xi_{J}\right):=\varphi\left(\xi_{1}, \ldots, \xi_{J}, 0 \ldots, 0\right)
$$

is a quasihomogeneous function of order $\ell(\gamma)$, i.e.

$$
\widehat{\varphi}_{\gamma}\left(t^{\beta_{1}} \xi_{1}, \ldots, t^{\beta_{J}} \xi_{J}\right)=t^{\ell(\gamma)} \widehat{\varphi}_{\gamma}\left(\xi_{1}, \ldots, \xi_{J}\right)
$$

where $\ell(\gamma)$ is defined by $\ell(\gamma)=\langle\alpha, \beta\rangle$ for a fixed $\beta \in \mathbb{Q}^{J},|\beta|=1$.

Therefore $\varphi_{\gamma}$ satisfies Euler's equality:

$$
\beta_{1} \xi_{1} \frac{\partial}{\partial \xi_{1}} \varphi_{\gamma}+\cdots \beta_{J} \xi_{J} \frac{\partial}{\partial \xi_{J}} \varphi_{\gamma}=\ell(\gamma) \varphi
$$

Hence, if there is some point $\xi^{*} \in \mathbb{R}^{J} \backslash 0$ such that $\nabla \varphi\left(\xi^{*}\right)=0$, then $\varphi\left(\xi^{*}\right)$ itself must also be zero. But this cannot take place because of the condition (3) of Definition 2.6. As the above argument can be applied to all the faces $\gamma \subset \Gamma(\varphi)$, strong Min-function must be non-degenerate.

Remark 2.10 Owing to this lemma, we can apply Theorem 2.5 to oscillatory integrals with a strong Min-function as the phase.

After these preparations we can state the following main theorem of this section.

Theorem 2.11 Assume that the homogeneous polynomial $F(\xi)$ satisfies the condition 2.8 and that the energy surface $S=\left\{\xi \in \mathbb{R}^{N} ; F(\xi)-1=0\right\}$ has only two points $( \pm 1,0, \ldots, 0)$ at which the normal to $S$ is parallel to $\omega_{0}=$ $(1,0, \ldots, 0)$. Then there exists $\delta>0$ small enough such that the following estimate holds for the fundamental solution of the operator $F(D)-I$ figured 
in (1.3). If $\left|\omega-\omega_{0}\right|<\delta$ with $\omega=x /|x|$

$$
|E(x)| \leq|x|^{-1 / d(\Gamma)}(\log |x|)^{\bar{\ell}(\Gamma)}
$$

where $d(\Gamma)$ denotes the height of the Newton's diagram of the following Newton polyhedron $\Gamma_{+}$:

$$
\begin{gathered}
\Gamma_{+}=\text {convex hull of }\left(\left\{\text { the Newton polyhedron of } f\left(\xi^{\prime}\right)=F\left(1, \xi^{\prime}\right)\right\}\right. \\
\left.\bigcup\left\{\left(\gamma_{2}, \ldots, \gamma_{N}\right) ;\left|\gamma_{2}+\cdots \gamma_{N}\right|>2 m\right\}\right) .
\end{gathered}
$$

The integer $\bar{\ell}(\Gamma)$ denotes the one introduced in (4) of Theorem 2.5. In other words, $E(x)$ decays asymptotically less slower than $E\left(x_{1}, 0\right)$ as $|x| \rightarrow \infty$ within a conical neighbourhood of the form $|x /| x\left|-\omega_{0}\right|<\delta$.

Proof. From Proposition 2.3, Theorem 2.5 and Lemma 2.9, we have the estimate (2.8) for $x=\left(x_{1}, 0, \ldots, 0\right)$. That we should observe the oscillatory integral with the phase $x_{1} \xi_{1}\left(\xi^{\prime}\right)$ can show this.

$$
\xi_{1}\left(\xi^{\prime}\right)=1-\frac{1}{2 m}\left\{\xi_{2}^{2 m}+\cdots+\xi_{N}^{2 m}+\sum_{\alpha \in \Gamma(f)} a_{j \alpha}{\xi^{\prime}}^{\alpha}\right\}+R\left(\xi^{\prime}\right)
$$

where the Newton Polyhedron of $R\left(\xi^{\prime}\right)$ is contained the set $\Gamma_{+}$:

$$
\begin{array}{r}
\Gamma_{+}=\text {convex hull of }\left(\left\{\text { the Newton polyhedron of } f\left(\xi^{\prime}\right)=F\left(1, \xi^{\prime}\right)\right\}\right. \\
\left.\bigcup\left\{\left(\gamma_{2}, \ldots, \gamma_{N}\right) ;\left|\gamma_{2}+\cdots \gamma_{N}\right|>2 m\right\}\right) .
\end{array}
$$

To show the estimate (2.8) for $x=\left(x_{1}, x_{2}, \ldots, x_{N}\right)$ with $x_{j} \neq 0$ at least for some $j \in\{2, \ldots, N\}$, firstly we will observe the case when $x_{2} \neq 0$ and $x_{j}=0$ for $3 \leq j \leq N$, because the estimate for this case contains essential points of the proof. The general situation will be explained after the proof of the case for $x=\left(x_{1}, x_{2}, 0, \ldots, 0\right)$. To see the asymptotic behaviour of $E\left(x_{1}, x_{2}, 0, \ldots, 0\right)$ it is enough to see that of $\Phi(0, x /|x|, x)$ (defined in (1.4) or (1.6) ) for $x=\left(x_{1}, x_{2}, 0, \ldots, 0\right)$. As it has been studied in the sketchy proof of Theorem 1.3, our center of interest will be the shape of the surface $S$ around the point $\stackrel{\circ}{\xi}=\left(\stackrel{\circ}{\xi}_{1}, \ldots, \stackrel{\circ}{\xi}_{N}\right)$ at which the normal to $S$ is parallel to $\omega=\left(x_{1} /|x|, x_{2} /|x|, 0, \ldots, 0\right)$. As it has been done in the proof of Theorem 1.3, we shall choose a suitable coordinate system $\left(\widetilde{\xi}_{1}, \widetilde{\xi}_{2}, \ldots, \widetilde{\xi}_{N}\right)$ such that the $\tilde{\xi}_{1}$-axis is parallel to $\omega$, in order to describe the surface $S$ in the form $\widetilde{\xi}_{1}=\widetilde{\xi}_{1}\left(\widetilde{\xi}_{2}, \ldots, \widetilde{\xi}_{N}\right)$. As the solution of the equation to determine the stationary points $\operatorname{grad} \xi_{1}\left(\xi^{\prime}\right)=\left(-1 / x_{1}\right)\left(x_{2}, \ldots, x_{N}\right)$ depends continuously 
on $\omega=x /|x|$, for every $\varepsilon>0$, there exists $\delta>0$ such that if $\left|\omega-\omega_{0}\right|<\delta$ then

$$
\left|\left(\stackrel{\circ}{\xi}_{1}, \stackrel{\circ}{\xi}_{2}, \ldots, \stackrel{\circ}{\xi}_{N}\right)-(1,0, \ldots, 0)\right|<\varepsilon
$$

Because of this continuity, we see that there exists small $\delta_{0}$ such that if $\left|\omega-\omega_{0}\right|<\delta_{0}$ then there exists only two points on $S$ at which the normal to $S$ is parallel to $\omega$, in view of the assumption imposed on the number of points on $S$ at which the normal to $S$ is parallel to $\omega_{0}$. If one of them is $\left(\stackrel{\circ}{\xi}_{1}, \ldots, \stackrel{\circ}{\xi}_{N}\right)$ the other has the form $\left(-\stackrel{\circ}{\xi}_{1}, \ldots,-\stackrel{\circ}{\xi}_{N}\right)$. We will study the situation around the point with $\stackrel{\circ}{\xi}_{1}>0$ (let us denote it by $\sigma^{+}(\omega)$ ) because the following argument is completely parallel for another point (denoted by $\left.\sigma^{-}(\omega)\right)$. Furthermore we assume that $\widetilde{\xi}_{1}(0)=\mu_{+}(\omega):=\left\langle\omega, \sigma^{+}(\omega)\right\rangle$ in the above coordinate system. As is seen from these setting of the situation, it is the oscillatory integral with phase $|x| \widetilde{\xi}_{1}\left(\widetilde{\xi}^{\prime}\right)$ that should be estimated. For this purpose it is enough to know the Newton diagram of $\widetilde{\xi}_{1}\left(\widetilde{\xi}^{\prime}\right) \in \mathbb{R}^{N-1}$. To see the degeneracy of this the coordinate change $\xi \rightarrow \widetilde{\xi}$.

$$
\left(\begin{array}{c}
\tilde{\xi}_{1} \\
\widetilde{\xi}_{2} \\
\vdots \\
\vdots \\
\widetilde{\xi}_{N}
\end{array}\right)=\left(\begin{array}{ccccc}
\omega_{1} & -\omega_{2} & & 0 & \\
\omega_{2} & \omega_{1} & & & \\
& & 1 & & \\
& & & \ddots & \\
& 0 & & & 1
\end{array}\right)\left(\begin{array}{c}
\xi_{1} \\
\xi_{2} \\
\vdots \\
\vdots \\
\xi_{N}
\end{array}\right)+\left(\begin{array}{c}
\varepsilon_{1} \\
\varepsilon_{2} \\
\vdots \\
\vdots \\
\varepsilon_{N}
\end{array}\right)
$$

where $\left(\varepsilon_{1}, \varepsilon_{2}, \ldots, \varepsilon_{N}\right)=\left(\stackrel{\circ}{\xi_{1}} \omega_{1}-\stackrel{\circ}{\xi}_{2} \omega_{2}-\mu_{+}(\omega),-\left(\stackrel{\circ}{\xi}_{1} \omega_{2}+\stackrel{\circ}{\xi}_{2} \omega_{1}\right), \stackrel{\circ}{\xi}_{3}, \ldots, \stackrel{\circ}{\xi}_{N}\right)$ denotes the shift between the line with direction $\omega$ passing through $\sigma(\omega)$ and the line with the same direction passing through the origin. Thus the function $\widetilde{\xi}_{1}\left(\widetilde{\xi}^{\prime}\right)$ of which behaviour we want to know around $\widetilde{\xi}^{\prime}=0$ is determined by the equation

$$
\begin{aligned}
F\left(\omega_{1}\left(\widetilde{\xi}_{1}-\varepsilon_{1}\right)+\omega_{2}\left(\widetilde{\xi}_{2}-\varepsilon_{2}\right),+\omega_{1}\left(\widetilde{\xi}_{2}-\varepsilon_{2}\right)-\omega_{2}\left(\widetilde{\xi}_{1}-\varepsilon_{1}\right),\right. \\
\left.\widetilde{\xi}_{3}-\stackrel{\circ}{\xi}_{3}, \ldots, \widetilde{\xi}_{N}-\stackrel{\circ}{\xi}_{N}\right)=1
\end{aligned}
$$

The claim to be shown is the following.

\section{Claim}

(The height $d(\Gamma)$ of the Newton diagram $\Gamma$ corresponding to $\Gamma_{+}$ of $(2.10))>($ the height of the Newton diagram 
of $\widetilde{\xi}_{1}\left(\widetilde{\xi}_{2}, \ldots, \widetilde{\xi}_{N}\right)$ as an analytic function germ at $\left.\widetilde{\xi}^{\prime}=0\right)$.

Proof of Claim.

We consider following two cases independently.

(1) There exist $3 \leq j_{1}, j_{2}, \ldots, j_{n} \leq N$ such that $\stackrel{\circ}{\xi}_{j_{1}} \cdots \stackrel{\circ}{\xi}_{j_{2}} \cdots \stackrel{\circ}{\xi}_{j_{n}} \neq 0$, and $\stackrel{\circ}{\xi}_{j}=0$ for $j \notin\left\{j_{1}, j_{2}, \ldots, j_{n}\right\}$.

(2) For all $j(3 \leq j \leq N), \stackrel{\circ}{\xi}_{j}=0$.

Case 1)

Under the above noted situation, we shall show that

$$
-\left(\frac{\partial}{\partial \xi_{j_{i}}}\right)^{2} \widetilde{\xi}_{1}>0 \quad \text { for } 1 \leq i \leq N .
$$

When the above inequalities are shown, we can directly conclude the following inclusion relation between the Newton polyhedra in question.

Newton polyhedron of $\widetilde{\xi}_{1}\left(\widetilde{\xi}_{2}, \ldots, \widetilde{\xi}_{N}\right)$

$$
\supset \text { convex hull of }\left(\bigcup_{1 \leq i \leq N}\left\{\left(0, \ldots, \stackrel{j_{i}}{2}, \ldots, 0\right)+\mathbb{R}^{N-1}\right\} \bigcup \Gamma_{+}\right) \supsetneqq \Gamma_{+} \text {. }
$$

This proves Claim in this case.

Let us begin to study the function germ $\left.\widetilde{(0}, \ldots, \widetilde{\xi}_{j_{1}}, \ldots, 0\right)$ of one variables with the following principal part,

$$
\begin{aligned}
& -\frac{1}{2 m} \sum_{\begin{array}{c}
\left(j, k, \ell, \alpha^{\prime \prime}\right) \in \Gamma_{+} \\
\alpha^{\prime \prime}=\left(\alpha_{j_{1}}, \ldots, \alpha_{j_{n}}\right)
\end{array}} a_{j k \ell \alpha^{\prime \prime}}\left(-\omega_{1} \varepsilon_{1}-\omega_{2} \varepsilon_{2}\right)^{j}\left(-\omega_{1} \varepsilon_{2}+\omega_{2} \varepsilon_{1}\right)^{k} \\
& \quad \times\left(\widetilde{\xi}_{j_{1}}+\stackrel{\circ}{\xi}_{j_{1}}\right)^{\ell} \stackrel{\circ}{\xi}_{j_{2}}^{\alpha_{j_{2}}} \ldots \stackrel{\circ}{\xi}_{j_{n}}^{\alpha_{j_{n}}}+\mu(\omega) .
\end{aligned}
$$

From Proposition 2.3, $1-\xi_{1}\left(\xi^{\prime}\right)$ is a strong Min-function and so is the function $\mu^{+}(\omega)-\widetilde{\xi}_{1}\left(0, \ldots, 0, \xi_{j_{1}}, \ldots, 0\right)$ (where $\xi_{j_{1}}=\widetilde{\xi}_{j_{1}}+\stackrel{\circ}{\xi}_{j_{1}}$ ) that is positive except the point $\xi_{j_{1}}=0$. From Bertini-Sard's theorem (see [AVG]) the points where Hessian of an analytic function of one variable vanish must be located in a discrete manner. Together with the positivity of $\mu^{+}(\omega)-\widetilde{\xi}_{1}\left(0, \ldots, \xi_{j_{1}}, \ldots, 0\right)$ except at the origin it is concluded that there 
exists small $\varepsilon_{j_{1}}>0$ such that for $0<\left|\xi_{j_{1}}\right|<\varepsilon_{j_{1}}$,

$$
-\left(\frac{\partial}{\partial \xi_{j_{1}}}\right)^{2} \widetilde{\xi}_{1}\left(0, \ldots, \xi_{j_{1}}, \ldots, 0\right)>0 .
$$

Therefore, if we take $\varepsilon=\varepsilon_{j_{1}}$ and choose some $\delta$ such that (2.11) holds, we see that for such $\delta$

$$
-\left(\frac{\partial}{\partial \xi_{j_{1}}}\right)^{2} \widetilde{\xi}_{1}\left(0, \ldots,-\dot{\circ}_{j_{1}}, \ldots, 0\right)>0 .
$$

For the other function germs $\widetilde{\xi}_{1}\left(0, \ldots, \xi_{j_{i}}, \ldots, 0\right)$ with $2 \leq i \leq n$, similar argument holds with other $\varepsilon_{j_{i}}$. Thus, if we set $\varepsilon=\min _{1 \leq i \leq n} \varepsilon_{j_{i}}$ and choose some $\delta$ anew such that (2.11) holds, we can conclude (2.12).

Case 2)

Further we suppose that $\stackrel{\circ}{\xi}_{3}=\cdots=\stackrel{\circ}{\xi}_{N}=0$.

In this case we shall observe the Newton polyhedron of the function germ $x_{1} \xi_{1}\left(\xi^{\prime}\right)+x_{2} \xi_{2}$ at its singular point $\left(\stackrel{\circ}{\xi}_{2}, 0, \ldots, 0\right)$. To see this, it is enough to check that there exists $\varepsilon>0$ such that if $0<\left|\xi_{j_{1}}\right|<\varepsilon$ then

$$
-\left(\frac{\partial}{\partial \xi_{2}}\right)^{2} \tilde{\xi}_{1}\left(\stackrel{\circ}{\xi}_{2}, 0, \ldots, 0\right)>0 .
$$

From the assumption that $-\xi_{1}\left(\xi^{\prime}\right)$ is a strong Min-function, we see that $-\xi_{1}\left(\xi_{2}, 0, \ldots, 0\right)$ is a strong Min-function of one variable $\xi_{2}$ and

$$
\left(\frac{\partial}{\partial \xi_{2}}\right)^{2} \widetilde{\xi}_{1}(0,0, \ldots, 0)=0 .
$$

From Theorem of Bertini-Sard and the fact that $\xi_{1}\left(\xi_{2}, 0, \ldots, 0\right)$ is a strong Min-function, we see that the required statement is obtained. Hence we have shown the following inclusion relation between Newton polyhedra

Newton polyhedron of $\widetilde{\xi}_{1}\left(\tilde{\xi}_{2}, \ldots, \widetilde{\xi}_{N}\right)$

$$
\supset \text { convex hull of }\left(\bigcup_{1 \leq i \leq N}\left\{(2,0, \ldots, 0)+\mathbb{R}_{+}^{N-1}\right\} \bigcup \Gamma_{+}\right) \supsetneqq \Gamma_{+} .
$$

The Claim is shown for $x=\left(x_{1}, x_{2}, 0, \ldots, 0\right)$.

In more general situations $x=\left(x_{1}, x_{2}, \ldots, x_{n}, 0, \ldots, 0\right)$, the proof for the case 1) can be achieved almost without modifications, while it concerns 
the positivity of the second derivative of a strong Min-function outside the origin.

As for case 2), we can show the positivity of $-\left(\frac{\partial}{\partial \xi_{j}}\right)^{2} \widetilde{\xi}_{1}(0),(2 \leq j \leq n)$ from the fact that $F\left(\xi_{1}, \xi_{2}, 0, \ldots, 0\right)$ is a Min-function with isolated local minimum together with Theorem of Bertini-Sard.

In general it is difficult to determine the Newton diagram of the function

$$
x_{1} \xi_{1}\left(\xi^{\prime}\right)+x_{2} \xi_{2}+\cdots+x_{N} \xi_{N}
$$

at it's singular point.

Assume that $\xi_{1}\left(\xi^{\prime}\right)$ is a germ with the Milnor number $\mu$, and the bases of its versal deformation are $\varphi_{1}\left(\xi^{\prime}\right), \ldots, \varphi_{\mu-N+1}\left(\xi^{\prime}\right), \xi_{2}, \ldots, \xi_{N}$. That is to say the versal deformation of $\xi_{1}\left(\xi^{\prime}\right)$ can be written as follows.

$$
\xi_{1}\left(\xi^{\prime}\right)+y_{1} \varphi_{1}\left(\xi^{\prime}\right)+\cdots+y_{\mu-N+1} \varphi_{\mu-N+1}\left(\xi^{\prime}\right)+x_{2} \xi_{2}+\cdots+x_{N} \xi_{N}
$$

where $\left(y_{1}, \ldots, y_{\mu-N+1}, x_{2}, \ldots, x_{N}\right) \in \mathbb{R}^{\mu}$. Therefore the bifurcation set of the deformation $x_{1} \xi_{1}\left(\xi^{\prime}\right)+x_{2} \xi_{2}+\cdots+x_{N} \xi_{N}$ can be regarded as the restriction of the big bifurcation set $\Sigma \subset \mathbb{R}^{\mu}$ of the deformation (2.13) restricted on the set $\left\{y_{1}=y_{2}=\cdots=y_{\mu-N+1}=0\right\}$. It also has a structure of a stratified set. This is the very situation that V.I. Arnol'd studied in [Ar].

He observed the topological metamorphoses (perestroika) that may happen with the intersection of the bifurcation set $\Sigma$ and a hypersurface $t(x)=t$ that is defined by a smooth Morsean function $t(x)$. Arnol'd calls this $t(x)$ "time function", because we get the pictures of stratified set $\Sigma_{t}=\left.\Sigma\right|_{t(x)=t}$ corresponding to each moment $t$. For the details one may consult [AVG] or [Z].

Let us define a series of sets $\sigma_{p, k} \subset \mathbb{R}^{N}, p \in \mathbb{Q}_{-}, k \in \mathbb{Z}_{+}$

$$
\begin{aligned}
\sigma_{p, k}= & \left\{\left(x_{1}, \ldots, x_{N}\right) ;|x /| x|-(1,0, \ldots, 0)|<\varepsilon \text { for some } \varepsilon>0,\right. \\
& \text { the oscillatory integral of the type }(2.6) \text { with the phase } \\
& |x| f\left(\xi^{\prime}\right)=x_{1} \xi_{1}\left(\xi^{\prime}\right)+x_{2} \xi_{2}+\cdots+x_{N} \xi_{N}(\tau=|x|) \\
& \text { can be dominated by } \left.|x|^{p}(\log |x|)^{k} \text { as }|x| \rightarrow \infty\right\} .
\end{aligned}
$$

According to Theorem 2.11,

$$
\sigma_{p, k} \supset \sigma_{p_{1}, k_{1}} \quad \text { if } p_{1}<p .
$$


Especially $\sigma_{p_{0}, k_{0}}$, with $p_{0}=-1 / d(\Gamma(f)), k_{0}=\bar{\ell}(\Gamma(f))$, has maximal codimension $N-1$ i.e. the line $\left(x_{1}, 0, \ldots, 0\right), x_{1} \neq 0$. As it has been remarked above, the set $\bigcup_{(p, k) \in\left(\mathbb{Q}_{-}, \mathbb{Z}_{+}\right)} \sigma_{p, k}$ is stratified. In general it is difficult to determine this structure of stratification, but in some special cases it is possible to know the strata.

Theorem 2.12 Assume that following two conditions are imposed on $F(\xi)$ besides condition 2.8 :

(1) All the coefficients $a_{j \alpha}$ are positive.

(2) For all $\alpha$ with $a_{j \alpha} \neq 0, \alpha \in(2 N)^{n-1}$.

Furthermore we assume that there exist only two points $( \pm 1,0, \ldots, 0)$ at which the normal to $S$ is parallel to $\omega_{0}=(1,0, \ldots, 0)$. Then there exists $\varepsilon>0$ small enough so that for the fundamental solution $E(x)$ determined by (1.3) and for $|x /| x|-(1,0, \ldots, 0)|<\varepsilon$, we have the following estimate that holds uniformly on the stratum

$$
\begin{aligned}
& \sigma_{I}=\left\{x \in \mathbb{R}^{N} ; x_{i} \neq 0 \text { for } i=1 \text { and } i \in I \subset\{1, \ldots, N\}\right. \\
& \left.\qquad \text { and } x_{j}=0 \text { for } j \notin I\right\} . \\
& |E(x)| \leq C(\omega)|x|^{-(I-1) / 2-1 / d(\Gamma(I))}(\log |x|)^{\bar{\ell}(\Gamma(I))}
\end{aligned}
$$

where $C(\omega)<\infty$ for each $\omega, 0<\left|\omega-\omega_{0}\right|<\varepsilon$, especially in the case when I contains single element except $1, I=\{1, i\}$, we have

$$
C(\omega) \approx\left(\frac{x_{i}}{|x|}\right)^{-(j(i)-2) / 2(j(i)-1)}, \quad \omega=\left(\frac{x_{1}}{|x|}, 0, \ldots, 0, \frac{\stackrel{i}{x_{i}}}{|x|}, 0, \ldots, 0\right)
$$

where

$\Gamma(I):\{\alpha=0$ for $i \in I\} \cap \Gamma$.

$j(i)$ : the intersection of the Newton diagram $\Gamma$ with $\alpha_{i}$-axis.

$d(\Gamma(I))$ : the height of the Newton diagram $\Gamma(I)$.

$\bar{\ell}(\Gamma(I))$ : the integer defined in Theorem 2.5, (4) for $\Gamma(I)$.

Proof. The domains in question on the surface $S$ are neighbourhoods of the points $( \pm 1,0, \ldots, 0)$. Among these two domains, we observe the part with $\xi>0$ only, as the situation is the same for another part. We proceed by means of an induction on the number of non-zero coordinates among $\left(x_{2}, \ldots, x_{N}\right) \in \mathbb{R}^{N-1}$. At first, let us study the case $I=\{1,2\}$, $x=\left(x_{1}, x_{2}, 0, \ldots, 0\right)$. Remark that the stationary point $\xi^{*}$ determined 
uniquely by the equations

$$
\begin{aligned}
& x_{1} \frac{\partial \xi_{1}}{\partial \xi_{2}}\left(\xi^{*}\right)+x_{2}=0, \\
& \frac{\partial \xi_{1}}{\partial \xi_{j}}\left(\xi^{*}\right)=0 \quad 3 \leq j \leq N
\end{aligned}
$$

has the form $\xi^{*}=\left(\xi_{2}^{*}, 0, \ldots, 0\right)$ with $\xi_{2}^{*} \neq 0$. This can be seen from the following argument. Owing to the condition $(2), \min \left\{\alpha_{j} ;\left(\alpha_{2}, \ldots, \alpha_{N}\right) \in\right.$ $\operatorname{supp} f\} \geq 2$. Therefore we get $x_{1} \frac{\partial \xi_{1}}{\partial \xi_{j}}\left(\xi_{2}, \ldots, \xi_{j-1}, \stackrel{j}{0}, \xi_{j+1}, \ldots, \xi_{N}\right)=0$.

On the other hand, as $\xi_{1}$ is a strong Min-function, there exists an even number $j(2)$ such that $\tilde{a}_{j(2) 0}>0$ with

$$
\frac{\partial \xi_{1}}{\partial \xi_{2}}\left(\xi^{*}\right)=\sum_{j \geq j(2)} j \widetilde{a}_{j 0}\left(\xi_{2}^{*}\right)^{j-1} .
$$

As for the uniqueness of the stationary point in the neighbourhood of $(1,0, \ldots, 0)$, from the conditions imposed on $f\left(\xi^{\prime}\right)$, we see that $\xi_{1}\left(\xi^{\prime}\right)$ is a concave function in some neighbourhood of $\xi^{\prime}=0$.

It is enough to remember the uniqueness of the point where the normal to $S$ is parallel to $\omega_{0}$, to see that the surface $S=\left(\xi_{1}\left(\xi^{\prime}\right), \xi_{2}, \ldots, \xi_{N}\right)$ has unique point at which $S$ is tangent to an affine hyperplane with normal $\omega=x /|x|$. After a coordinate change $\widetilde{\xi}_{2}=\xi_{2}-\xi_{2}^{*}$, we have the following principal part with Morsean type term with respect to $\widetilde{\xi}_{2}$

$$
\begin{aligned}
& \text { the principal part of }-\xi_{1}\left(\widetilde{\xi}_{2}+\xi_{2}^{*}, \xi^{\prime \prime}\right)-x_{2} \widetilde{\xi}_{2} / x_{1} \\
& =\widetilde{a}_{20} \widetilde{\xi}_{2}^{2}+\sum_{\gamma \in\{\alpha=0\} \cap \Gamma} a_{j \gamma} \xi_{2}^{* j} \xi^{\prime \prime \gamma}
\end{aligned}
$$

with $\tilde{a}_{20}>0$. This can be proved by a similar argument as case 2 ) in Theorem 2.11.

The right hand side has "the convex hull of $\{(2,0, \ldots, 0)\} \cup\left(\Gamma \cap\left\{\alpha_{2}=\right.\right.$ $0\})$ " as its Newton polyhedron. In view of Theorem 2.5, this fact yields (2.14) on the stratum $\left\{x \in \mathbb{R}^{N} ; x_{1} x_{2} \neq 0, x_{j}=0(3 \leq j \leq N)\right\}$. As for the estimate of $C\left(\omega_{2}, 0\right)$, we begin by determining $\xi_{2}^{*}$ from the following equation.

$$
x_{1} \frac{\partial \xi_{1}}{\partial \xi_{2}}\left(\xi_{2}^{*}\right)+x_{2}=x_{1} \sum_{j \geq j(2)} j \widetilde{a}_{j 0} \xi_{2}^{* j-1}+x_{2}=0 .
$$


So

$$
\xi_{2}^{*}=\left(x_{2} / j(2) a_{j(2) 0} x_{1}\right)^{1 /(j(2)-1)}+O\left(x_{2}^{2 /(j(2)-1)}\right) .
$$

As the oscillatory integral to be estimated has the form

$$
e^{i x_{2} \xi_{2}^{*}} \int e^{i x_{1} \xi_{1}\left(\xi_{2}^{*}, \xi^{\prime \prime}\right)} d \xi^{\prime \prime} \int e^{i x_{1} \widetilde{a}_{20} \widetilde{\xi}_{2}^{2}} \varphi\left(\widetilde{\xi}_{2}, \xi^{\prime \prime}\right) d \widetilde{\xi}_{2}
$$

it remains to calculate the coefficient to give the estimate. In fact the needed value can be gotten as follows,

$$
\begin{aligned}
\tilde{a}_{20}= & \left(\frac{\partial}{\partial \xi_{2}}\right)^{2} \xi_{1}\left(\xi_{2}^{*}, 0\right)=\sum_{j \leq j(2)} j(j-1) \widetilde{a}_{j 0} \xi_{2}^{* j-2} \\
= & j(2)(j(2)-1) a_{j(2) 0}\left(x_{2} / j(2) a_{j(2) 0} x_{1}\right)^{(j(2)-2) /(j(2)-1)} \\
& \quad+O\left(x_{2}^{j(2)-1 /(j(2)-1)}\right) .
\end{aligned}
$$

By applying the stationary phase method to phases with Morsean singularities, we obtain the required estimate.

For the strata $\sigma_{I}$ with $|I|>2,\{1,2\} \in I$, we have only to show that

$$
\begin{aligned}
& \text { the Newton polyhedron of the phase } i\left(x_{1} \xi_{1}\left(\xi^{\prime}\right)+\sum_{j \in I} x_{j} \xi_{j}\right) \\
& =\text { convex hull of } \bigcup_{j \in I}\{(0, \ldots, 0, \check{2}, 0, \ldots, 0)\} \bigcup\left\{\Gamma \cap\left\{\alpha_{j}=0, j \in I\right\}\right\} .
\end{aligned}
$$

This can be proved from the fact that this phase has the stationary point $\xi^{*} \in\{\xi \neq 0$ for $j \in I$ and $\xi=0$ for $i \notin I\}$ and the argument similar to that of case 2) in Theorem 2.11.

\section{Estimates of the fundamental solutions of 4-th order elliptic operators in two independent variables}

In this section our aim is to obtain asymptotic behaviour of the fundamental solution $E(x)$ of the equation $(0.2)$ with $F\left(D_{1}, D_{2}\right)=D_{1}^{4}+$ $p D_{1}^{2} D_{2}^{2}+q D_{1} D_{2}^{3}+r D_{2}^{4}$. Evidently all 4 -th order elliptic operators on $\mathbb{R}^{2}$ can be written in this form after linear change of coordinates. For this purpose, our main task will be to classify the singular points on the curve $S=\left\{\left(\xi_{1}, \xi_{2}\right) \in \mathbb{R}^{2} ; F\left(\xi_{1}, \xi_{2}\right)=1\right\}$. To establish the relationship between the vanishing of the Hessian of $F(\xi)$ and that of the curvature, we prepare the following lemma. 
Lemma 3.1 The curvature of the curve $S=\left\{\left(\xi_{1}, \xi_{2}\right) \in \mathbb{R}^{2} ; F\left(\xi_{1}, \xi_{2}\right)=1\right\}$ vanishes at a point $\stackrel{\circ}{\xi} \in S$ if and only if the Hessian of the function $F(\xi)$ vanishes at this point.

Furthermore if we describe the curve $S$

$$
G\left(\xi_{1}, \xi_{2}\right)=\xi_{1}^{* 2}-\left[\stackrel{\circ}{\xi}_{1}^{* 2}+g\left(\xi_{2}{ }^{*}-\stackrel{\circ}{\xi}_{2}^{*}\right)^{m}+O\left(\left(\xi_{2}^{*}-{\stackrel{\circ}{\xi_{2}}}^{*}\right)^{m+1}\right)\right]=0
$$

in a coordinate system $\left(\xi_{1}^{*}, \xi_{2}^{*}\right)$ obtained by a rotation around the origin from $\left(\xi_{1}, \xi_{2}\right)$ and the expression $\left(\stackrel{\circ}{\xi}_{1}^{*}, \stackrel{\circ}{\xi}_{2}{ }^{*}\right)$ for the point $\stackrel{\circ}{\xi}^{*}$ in $\left(\xi_{1}^{*}, \xi_{2}^{*}\right)$-coordinate system, then the curvature $K$ has the following form

$$
\begin{aligned}
K=-g m & (m-1)\left(\xi_{2}^{*}-\stackrel{\circ}{\xi}_{2}^{*}\right)^{m-2}+O\left(\left(\xi_{2}^{*}-\stackrel{\circ}{\xi}_{2}^{*}\right)^{m-1}\right) / \\
& \left.\left.\quad \dot{\circ}_{1}^{* 2}+\left[g m\left(\xi_{2}^{*}-\stackrel{\circ}{\xi}_{2}^{*}\right)^{m-1}\right]^{2}\right)\right\}^{3 / 2}+O\left(\left(\xi_{2}^{*}-\stackrel{\circ}{\xi}_{2}^{*}\right)^{m-1}\right)
\end{aligned}
$$

in the neighbourhood of the point $\stackrel{\circ}{\xi}$.

Therefore the curvature of $S$ has a zero of order $(m-2)$ provided that the Hessian of the function $F$ has zero of order $(m-2)$ at that point.

Proof. As it is well-known that for curves defined as the zero points of a function $F\left(\xi_{1}, \xi_{2}\right)$ of which gradient never vanishes, its curvature can be written in the following way:

$$
K=\frac{F_{11} F_{2}^{2}-2 F_{12} F_{1} F_{2}+F_{22} F_{1}^{2}}{\left(F_{1}^{2}+F_{2}^{2}\right)^{3 / 2}}
$$

where $F_{j}=\frac{\partial F}{\partial \xi_{j}}$ and $F_{i j}=\frac{\partial^{2} F}{\partial \xi_{i} \partial \xi_{j}}$. We see that the denominator of this expression can be interpreted as a quadratic form depending on the variables $\xi$.

$$
\left(\begin{array}{ll}
F_{2} & -F_{1}
\end{array}\right)\left(\begin{array}{ll}
F_{11} & F_{12} \\
F_{12} & F_{22}
\end{array}\right)\left(\begin{array}{c}
F_{2} \\
-F_{1}
\end{array}\right)
$$

Let us name this expression $L(\xi)$. The quadratic form $L(\xi)$ may vanish either 1) Hess $F$ has indefinite signature, or 2) det Hess $F$ vanishes.

We show that case 1) cannot take place.

Assume that we want to investigate the situation around the point $\stackrel{\circ}{\xi} \in S$. Owing to Lemma 1.1, we have a factorization of the following form

$$
F(\xi)-1=G(\xi) T(\xi)
$$


where $G=0$ on $S$ and $T \neq 0$ on $S$. Because of the analyticity of $G(\xi)$ in $\left(\xi_{1}^{*}, \xi_{2}^{*}\right)$ in the neighbourhood of $\stackrel{\circ}{\xi}$, we can choose a special coordinate system $\left(\xi_{1}^{*}, \xi_{2}^{*}\right)$ obtained by a rotation around the origin such that $G$ takes the form like (3.1). If case 1) takes place, then the function $G$ must have the form

$$
G\left(\xi_{1}\left(\xi^{*}\right), \xi_{2}\left(\xi^{*}\right)\right)=\xi_{1}^{* 2}-\left\{\stackrel{\circ}{\xi}_{1}^{* 2}+g\left(\xi_{2}^{*}-\stackrel{\circ}{\xi}_{2}^{*}\right)^{2}+O\left(\left(\xi_{2}^{*}-\stackrel{\circ}{\xi}_{2}^{*}\right)^{3}\right)\right\}
$$

with $g>0$. Therefore in the neighbourhood of the point $\stackrel{\circ}{\xi}$, we have a non-vanishing expression

$$
L\left(\xi_{1}\left(\xi^{*}\right), \xi_{2}\left(\xi^{*}\right)\right)=-g\left(2{\stackrel{\circ}{\xi_{1}}}^{*}\right)^{2}+2 g\left(\xi_{2}^{*}-\stackrel{\circ}{\xi}_{2}^{*}\right)^{2}+O\left(\left(\xi_{2}^{*}-\stackrel{\circ}{\xi}_{2}^{*}\right)^{3}\right)
$$

that is evidently negative at $\xi^{*}=\stackrel{\circ}{\xi}$.

So we think of the case 2). In the same manner, we may assume that $S$ is given by

$$
G\left(\xi_{1}\left(\xi^{*}\right), \xi_{2}\left(\xi^{*}\right)\right)=0 .
$$

It is easy to see that

$$
\begin{aligned}
& \operatorname{det} \operatorname{Hess}_{\xi^{*}} G\left(\xi_{1}\left(\xi^{*}\right), \xi_{2}\left(\xi^{*}\right)\right) \\
& \quad=2 g m(m-1)\left(\xi_{2}^{*}-\stackrel{\circ}{\xi}_{2}^{*}\right)^{m-2}+O\left(\left(\xi_{2}^{*}-\stackrel{\circ}{\xi}_{2}^{*}\right)^{m-1}\right) .
\end{aligned}
$$

Owing to the fact that det Hess $G$ is invariant under the rotation of coordinates, i.e. det $\operatorname{Hess}_{\xi^{*}} G\left(\xi_{1}\left(\xi^{*}\right), \xi_{2}\left(\xi^{*}\right)\right)=\operatorname{det} \operatorname{Hess}_{\xi} G\left(\xi_{1}, \xi_{2}\right)$, we see that vanishing order of the curvature coincides with that of $\operatorname{det} \operatorname{Hess}_{\xi} G\left(\xi_{1}, \xi_{2}\right)$ at $\stackrel{\circ}{\xi}$.

Thus the problem to know where the curvature of the curve $S$ vanishes is reduced to the problem to know the location of the zeros of the function $\operatorname{det} \operatorname{Hess}_{\xi} G\left(\xi_{1}, \xi_{2}\right)$. It is easy to see the following after direct computation.

Lemma 3.2 For $F(\xi)=\xi_{1}^{4}+p \xi_{1}^{2} \xi_{2}^{2}+q \xi_{1} \xi_{2}^{3}+r \xi_{2}^{4}$, we have

$$
\begin{gathered}
H(\xi):=\operatorname{det} \operatorname{Hess}_{\xi} F(\xi) \\
=24 p \xi_{1}^{4}+72 q \xi_{1}^{3} \xi_{2}+12\left(12-p^{2}\right) \xi_{1}^{2} \xi_{2}^{2} \\
\quad-12 p q \xi_{1} \xi_{2}^{3}+\left(24 p r-9 q^{2}\right) \xi_{2}^{4}
\end{gathered}
$$

The classification of the singular points (the points where the curvature 
vanishes) on $S$ is transformed into the classification of the homogeneous polynomials $H(\xi)$, that shall be done in terms of the coefficients $(p, q, r) \in$ $\mathbb{R}^{3}$.

Definition 3.3 We call a point $(p, q, r) \in \mathbb{R}^{3}$ admissible if and only if $F(\xi)=\xi_{1}^{4}+p \xi_{1}^{2} \xi_{2}^{2}+q \xi_{1} \xi_{2}^{3}+r \xi_{2}^{4}>0$ for all $\xi \in \mathbb{R}^{3} \backslash\{0\}$.

The domain consisting of all admissible points is called admissible domain.

Remark 3.4 If $(p, q, r)$ belongs to the admissible domain, then $r$ must de strictly positive. Therefore the admissible domain is contained in the half space $\left\{(p, q, r) \in \mathbb{R}^{3} ; r>0\right\}$.

For the sake of simplicity, we write down the function $H(\xi)$ in the following form

$$
\begin{aligned}
& H(\xi)=24 p\left(\xi_{1}^{\prime 4}+P{\xi_{1}^{\prime}}^{2}{\xi_{2}^{\prime}}^{2}+Q \xi_{1}^{\prime}{\xi_{2}^{\prime}}^{3}+R{\xi_{2}^{\prime}}^{4}\right) \\
& \text { where } \xi_{1}^{\prime}=\xi_{1}+\frac{q}{p} \xi_{2} \\
& \xi_{2}^{\prime}=\xi_{2} \\
& P=\left[2^{2} p\left(2^{2} 3 r-p^{2}\right)-3^{3} q^{2}\right] / 2^{3} p^{2} \\
& Q=\left[(3 q)^{3}-2^{5} 3 q p\left(2^{2} 3 r-p^{2}\right)\right] / 2^{3} p^{3}-q / 2 \\
& R=\left[3^{2}\left(2^{2} 3 r-p^{2}\right) q^{2}\right] / 2^{5} p^{3}-3^{5} q^{4} / 2^{5} p^{3}-3 q^{2} / 2^{3} p+r \text {. }
\end{aligned}
$$

Further to judge when the polynomial $H(\xi)$ may have multiple factors, we study the image of the admissible domain by the mapping $\Psi:(p, q, r) \mapsto$ $(P, Q, R)$. As this mapping is defined only for $p \neq 0$, we restrict ourselves to this case when we think of the mapping $\Psi$. Before to mention our main statement in this section, we shall introduce the notion of a swallow's tail that plays important role in singularity theory.

\section{Lemma 3.5}

(1) The swallow's tail is assimilated to a surface that consists of the points $(P, Q, R)$ for which the equation $x^{4}+P x^{2}+Q x+R=0$ has multiple roots.

(2) It can be parametrized as

$$
R=3 t^{4}+P t^{2}
$$




$$
Q=-4 t^{3}-2 P t, \quad t \in \mathbb{R}
$$

(3) The polynomial with coefficients $(P, Q, R)$ situated in the domain $(n)$ have $n$ real roots, $n=0,2$ or 4 . Further we will use the naming "Domain $(n)$ ".

(4) The polynomials corresponding to the generic points on the swallow's tail have a double root.

(5) The two curves $($ in $R<0)$ issued from the origin named "back bones" (after V.P. Palamodov's naming), correspond to the polynomials with triple roots. It is given by a one parameter expression:

$$
(P, Q, R)=\left(-6 t^{2}, 8 t^{3},-3 t^{4}\right), \quad t \in \mathbb{R} .
$$

(6) Another curve $(R>0)$ issued from the origin is called the Maxwell set. It can be given by,

$$
\begin{aligned}
& R=P^{2} / 4 \\
& Q=0 .
\end{aligned}
$$

The polynomials corresponding to the points on this curve have two double roots.

Proposition 3.6 For $p \neq 0$, the Hessian $H(\xi)$ has

(1) no roots if $\psi(p, q, r) \in \operatorname{Domain}(0)$,

(2) four distinct simple roots if $\psi(p, q, r) \in \operatorname{Domain}(4)$,

(3) two distinct simple roots and one double root if $\psi(p, q, r)$ falls on the generic position of the swallow's tail,

(4) two distinct simple roots if $\psi(p, q, r) \in \operatorname{Domain}(2)$,

(5) two distinct double roots if $\psi(p, q, r) \in$ Maxwell set,

(6) There is no admissible point $(p, q, r)$ such that $\psi(p, q, r) \in$ "back bone". Here the roots of the Hessian means

$$
\text { \{roots of } \left.H\left(\xi_{1}, 1\right)=0\right\} \bigcup\left\{\text { roots of } H\left(1, \xi_{2}\right)=0\right\} / \sim
$$

where the equivalence relation $\sim$ shall be understood as " $\xi_{1}^{*} \sim \xi_{2}^{*}$ iff $\xi_{1}^{*}=$ $1 / \xi_{2}^{*} "$

Before coming into the proof of this Proposition, we will formulate the main theorem of this section.

Theorem 3.7 We have the following five types of the asymptotic behaviour of the fundamental solution to the 4-th order elliptic operator with coeffi- 
cients

$$
F\left(D_{1}, D_{2}\right)-I=D_{1}^{4}+p D_{1}^{2} D_{2}^{2}+q D_{1} D_{2}^{3}+r D_{2}^{4}-I
$$

corresponding to the cases (1)-(5) in Proposition 3.6.

(1) If $\psi(p, q, r) \in \operatorname{Domain}(0)$, then the fundamental solution $E(x)$ has the asymptotic expansion modulo rapidly decreasing smooth functions like in (1.5) for $x=r \omega$ for all $\omega \in S^{1}$.

$$
E(x) \sim \sum_{j=1}^{2} e^{-i \mu_{j}(\omega) r} \sum_{s=0}^{\infty} a_{j s}(\omega) r^{-1 / 2-s}
$$

where $a_{j 0}=(2 \pi)^{1 / 2}\left|\kappa_{j}(\omega)\right|^{-1 / 2} f\left(\sigma^{j}(\omega)\right) e^{-\gamma_{j} \pi / 4} \kappa_{j}:$ the curvature of $S$ at the point $\sigma^{j}(\omega) \cdot \mu_{j}=\left\langle\sigma^{j}(\omega), \omega\right\rangle$ : the projection of the point $\sigma^{j}(\omega)$ on the line parallel to $\omega$ passing through the origin. $\gamma_{j}$ : inertia index of the curvature form at $\sigma^{j}(\omega)$.

(2) If $\psi(p, q, r) \in \operatorname{Domain}(4)$, then $E(x)$ has the asymptotic expansion modulo rapidly decreasing smooth functions

$$
E(x) \sim-i(2 \pi)^{-1} e^{ \pm i \mu\left(-\xi_{j}^{*}\right) r} \sum_{s=0}^{\infty} a_{j s}( \pm \omega) r^{(-1-s) / 3}
$$

on the eight half lines $x= \pm r \omega_{j}, j=1, \ldots, 4$, where

$$
\begin{aligned}
& a_{j 0}( \pm \omega)=\frac{1}{3\left|\nabla F\left(\xi_{j}^{*}\right)\right|} \Gamma(1 / 3)\left(1 / g_{j}\right)^{1 / 3} e^{\pi i / 6} \\
& g_{j}=\left.\frac{1}{2 \cdot 3 !}\left\{\frac{1}{\left|\nabla F\left(\xi_{j}^{*}\right)\right|}\left(-F_{1}\left(\xi_{j}^{*}\right) \frac{\partial}{\partial \xi}+F_{2}\left(\xi_{j}^{*}\right) \frac{\partial}{\partial \xi}\right)\right\}^{3} G\left(\xi_{1}, \xi_{2}\right)\right|_{\xi=\xi_{j}^{*}} \\
& \mu\left( \pm \xi_{j}^{*}\right)=\frac{4 F\left(\xi_{j}^{*}\right)}{\left|\nabla F\left(\xi_{j}^{*}\right)\right|} \quad j=1, \ldots, 4 .
\end{aligned}
$$

Notions that are not specified here are those that have already been used in the former part of this paper.

On the lines out of these directions (i.e. $x=r \omega, \omega \neq \omega_{j}, j=$ $1, \ldots, 4) E(x)$ has the asymptotic behaviour like (3.3).

(3) If $\psi(p, q, r)$ falls on the generic position of the swallow's tail, then $E(x)$ has the asymptotic expansion modulo rapidly decreasing smooth 
functions

$$
E(x) \sim-i(2 \pi)^{-1} e^{-i \mu\left( \pm \xi_{j}^{*}\right) r} \sum_{s=0}^{\infty} a_{j s}( \pm \omega) r^{(-1-s) / 4}
$$

on two half lines $x= \pm r \omega_{3}$.

$$
\begin{aligned}
& a_{j 0}( \pm \omega)=\frac{1}{4\left|\nabla F\left(\xi_{j}^{*}\right)\right|} \Gamma(1 / 4)\left(1 / g_{j}\right)^{1 / 4} e^{\pi i / 8} \\
& g_{j}=\left.\frac{1}{2 \cdot 4 !}\left\{\frac{1}{\left|\nabla F\left(\xi_{j}^{*}\right)\right|}\left(-F_{1}\left(\xi_{j}^{*}\right) \frac{\partial}{\partial \xi}+F_{2}\left(\xi_{j}^{*}\right) \frac{\partial}{\partial \xi}\right)\right\}^{4} G\left(\xi_{1}, \xi_{2}\right)\right|_{\xi=\xi_{j}^{*}}
\end{aligned}
$$

There are four half lines $x= \pm r \omega_{j}, j=1,2$, on which the asymptotic expansion like (3.4) holds.

On the line out of these directions (i.e. on the lines $x= \pm r \omega_{j}$, $j=1,2,3)$ it has the asymptotic behaviour like (3.3).

(4) If $\psi(p, q, r) \in \operatorname{Domain}(2)$, then there are four half lines $x= \pm r \omega_{j}, j=$ 1,2 on which the asymptotic expansion like (3.4) holds.

On the lines out of these directions it has the asymptotic behaviour like (3.3)

(5) If $\psi(p, q, r) \in$ Maxwell set, then there are four half lines $x= \pm r \omega_{j}, j=$ 1,2 on which the asymptotic expansion like (3.5) holds on the lines out of (3.3).

Proof of Theorem 3.7. We shall estimate the integral

$$
\int_{V_{j}} \frac{\varphi_{j}(\xi) e^{-i r \omega \xi}}{T(\xi)|\nabla G(\xi)|} d S
$$

where $V_{j} \subset S(j=1,2)$ contains only one point $\sigma^{j}(\omega)$ where the normal to $S$ is parallel to $\omega$.

In the neighbourhood of the stationary point $\sigma^{j}(\omega)=\left(\stackrel{\circ}{\xi_{1}^{*}}, \stackrel{\circ}{\xi_{2}^{*}}\right)$, we can take a suitable coordinate system $\left(\xi_{1}^{*}, \xi_{2}^{*}\right)$ so that the curve $S$ should be expressed as in (3.1).

By the aid of this coordinate change the phase of the integrand (3.6) can be written as follows.

$$
-i r\left\{\frac{\omega_{1} F_{1}(\sigma(\omega))+\omega_{2} F_{2}(\sigma(\omega))}{|\nabla F(\sigma(\omega))|} \xi_{1}^{*}+\frac{-\omega_{1} F_{2}(\sigma(\omega))+\omega_{2} F_{1}(\sigma(\omega))}{|\nabla F(\sigma(\omega))|} \xi_{2}^{*}\right\} .
$$

Case 1) In view of Lemma 3.1, there is no point where $H(\xi)$ vanishes 
on $S$. Therefore the same results as in Theorem 1.3 holds.

Case 2) Taking into account Lemma 3.1 and Proposition 3.6, there exist eight points on $S$ where the curvature vanishes for the first order.

Let us take one of these points $\sigma(\omega)=\left(\stackrel{\circ}{\xi}_{1}^{*}, \stackrel{\circ}{\xi}_{2}^{*}\right)$ at which the normal to $S$ is parallel to $\omega$.

As $\left(\omega_{1}, \omega_{2}\right)=\left(\frac{F_{1}(\sigma(\omega))}{|\nabla F(\sigma(\omega))|}, \frac{F_{2}(\sigma(\omega))}{|\nabla F(\sigma(\omega))|}\right)$, the phase will take form

$$
-i r\left(\stackrel{\circ}{\xi}_{1}^{*}+\frac{g_{j}}{2}\left[\xi_{2}^{*}-\stackrel{\circ}{\xi}_{2}\right]^{3}+R\left(\xi_{2}^{*}\right)\right)
$$

with $R_{4}=$ terms higher than 4 -th order.

So (3.6) becomes

$$
\int_{V_{j}} \frac{\varphi_{j}\left(\xi\left(\xi^{*}\right)\right)}{T\left(\xi\left(\xi^{*}\right)\right)\left|\nabla G\left(\xi\left(\xi^{*}\right)\right)\right|} \exp \left[-i r\left(\stackrel{\circ}{\xi}_{1}^{*}+\frac{g_{j}}{2}\left[\xi_{2}^{*}-\stackrel{\circ}{\xi_{2}}\right]^{3}+R\left(\xi_{2}^{*}\right)\right)\right] d \xi_{2}^{*} .
$$

To calculate this integral we use the formula by Erdélyi [E] in spite of Theorem 2.6 due to Varchenko.

\section{Erdélyi's formula}

$$
\begin{aligned}
& \int_{\alpha}^{\beta} e^{i r g(\eta)} h(\eta) d \eta \\
& =e^{i r g(\gamma)} \sum_{s=0}^{N-1} \frac{\kappa^{(n)}(\gamma)}{s ! \rho} \Gamma\left(\frac{s+\lambda}{\rho}\right) \exp \left(\frac{2 \pi i(s+\lambda)}{2 \rho}\right) r^{-(s+\lambda) / \rho} \\
& \quad+O\left(r^{-(N+\lambda) / \rho}\right) \quad \text { as } r \rightarrow \infty
\end{aligned}
$$

where $g(\eta)$ is a smooth function

$$
\begin{aligned}
& g(\eta)=g(\gamma)+(\eta-\gamma)^{\rho} g_{1}(\eta), \quad \rho>1 \\
& h(\eta)=(\eta-\gamma)^{\lambda-1} h_{1}(\eta) \\
& \kappa^{(n)}(\eta) \text { is a function determined by } h \text { and } h_{1}, \text { where } \\
& \kappa^{(0)}=h_{1}(\gamma) .
\end{aligned}
$$

In our case

$$
\begin{aligned}
& g\left(\xi_{2}^{*}\right)=\stackrel{\circ}{\xi}_{1}^{*}+\frac{g_{j}}{2}\left(\xi_{2}^{*}-\stackrel{\circ}{\xi}_{2}^{*}\right)^{3}+R_{4}\left(\xi_{2}^{*}\right), \quad \rho>1 \\
& h\left(\xi_{2}^{*}\right)=\left|\nabla G\left(\xi\left(\xi^{*}\right)\right)\right|^{-1} T\left(\xi^{*}\right)^{-1}=\left|\nabla F\left(\xi\left(\xi^{*}\right)\right)\right|^{-1} .
\end{aligned}
$$

It is enough to remark that

$$
\frac{\partial}{\partial \xi_{2}^{*}}=\frac{1}{|\nabla F(\stackrel{\circ}{\xi})|}\left(-F_{1}(\stackrel{\circ}{\xi}) \frac{\partial}{\partial \xi_{2}}+F_{2}(\stackrel{\circ}{\xi}) \frac{\partial}{\partial \xi_{1}}\right)
$$


in the ancient coordinate system, in order to show that

$$
g_{j}=\left.\frac{1}{3 !}\left\{\frac{1}{|\nabla F(\stackrel{\circ}{\xi})|}\left(-F_{1}(\stackrel{\circ}{\xi}) \frac{\partial}{\partial \xi_{2}}+F_{2}(\stackrel{\circ}{\xi}) \frac{\partial}{\partial \xi_{1}}\right)\right\}^{3} G(\xi)\right|_{\xi=\stackrel{\circ}{\xi}} .
$$

Thus we have calculated all the quantities that appears in (3.4).

At last, the integration around the point at which the outer normal to $S$ is directed to $-\omega$ survives among the contributions to $E(r \omega)$ while (1.3) and Lemma 1.2 shall be taken into account.

For the other cases the integral can be calculated completely in the similar way as in case 2), except the situation that there may paper points where the curvature vanishes for 2 nd order.

Proof of Proposition 3.6. The statements (1)-(5) are clearly seen in view of Lemma 3.5. What we shall see is that the positivity of $F(\xi)$ for such $(p, q, r)$ that $\psi(p, q, r) \in$ "back bones" can not take place.

First of all, we should remark that it is enough to grasp the location of the back bones and the image only in a small neighbourhood of the origin.

One has only to check the invariance of the image of the admissible domain with respect to translation $(p, q, r) \mapsto\left(s^{2} p, s^{3} q, s^{4} r\right)$ i.e.

$$
\begin{aligned}
& P\left(s^{2} p, s^{3} q, s^{4} r\right)=s^{2} P(p, q, r) \\
& Q\left(s^{2} p, s^{3} q, s^{4} r\right)=s^{3} Q(p, q, r) \\
& R\left(s^{2} p, s^{3} q, s^{4} r\right)=s^{4} R(p, q, r) \quad \text { for all } s>0,
\end{aligned}
$$

and to see the fact that if $(P, Q, R) \in$ one of strata of swallow's tail then $\left(s^{2} P, s^{3} Q, s^{4} R\right)$ also belongs to the same stratum. As we know that the admissible domain is contained in $r>0$, we aim to figure out the image of the hyperplane $r=0$ by then mapping $\psi$. For $r>0$, we have

$$
\begin{aligned}
& P=-p / 2-(3 / 2)^{3} q^{3} / p^{2} \\
& Q=(3 / 2)^{3} q^{3} / p^{3}+23 q / 2 \\
& R=-3 \cdot 7 q^{2} / 2^{5} p-3^{5} q^{4} / 2^{8} p^{4} .
\end{aligned}
$$

Let us denote by $\gamma$ the intersection of the surface $\psi(\{r=0\})$ and the parabola $R=-P^{2} / 12$ in order to see the disposition of $\gamma$ and the back bones, while the back bones lie on the parabola $R=-P^{2} / 12$. As it is seen from (3.2) the surface $\psi(\{r=0, p<0\})$ cannot have intersection with back bones. For an instant, we will observe the cases $p>0$ only. 
By substituting (3.2) to the equation $R=-P^{2} / 12$, we get $P^{3}=3^{2} \cdot 5 q^{2}$ that designates the preimage $\psi^{-1}(\gamma)$. In other words, the curve $\gamma$ can be described in $(P, Q, R)$-space by using $p>0$ as a parameter.

$$
\begin{aligned}
& P=-\left(1 / 2+3 / 2^{3} \cdot 5\right) p \\
& Q=1 / 2 \sqrt{5}\left(1 / 2^{2} \cdot 5+23 / 3\right) p^{3 / 2} \\
& R=-1 / 2^{5} \cdot 5\left(7 / 3+3 / 2^{3} \cdot 5\right) p^{2} .
\end{aligned}
$$

This means that the projection of this curve on $(P, Q)$-plane has the form

$$
Q^{2}=(2 / 23)^{3 / 2} 463 \cdot P^{3} / 3 .
$$

Meanwhile the projection of the back bone has the form

$$
Q^{2}=(2 / 3)^{3} P^{3} .
$$

By comparing these two expressions, the openness of the cusp $\gamma$ is bigger than that of a cusp formed by the back bones. Hence we come to know the disposition of these two cusps on a parabola.

Further we think of the curve $\gamma_{t}$ that is obtained as the intersection of $\psi(\{r=0\})$ and the parabola $\Pi_{t}=\left\{(P, Q, R) ; R=-t P^{2}, t>0\right\}$. As is easily seen, the distance between parabola $\Pi_{t}$ and the plane $R=0$ tends to zero in the neighbourhood of the origin when $t$ tends to zero. After a messy calculation, we can conclude that the cusp $\gamma_{t}$ tends to the negative $P$-axis in decreasing its openness as a cusp, as $t$ tends to zero. This can be seen from the following parametrization:

$$
\begin{aligned}
& P=-c_{1} p / t+O\left(t, p^{2}\right) \\
& Q=c_{2} p^{3 / 2} / \sqrt{t}+O\left(t, p^{2}\right) \\
& R=-c_{3} p^{2} / t+O\left(t, p^{3}\right) .
\end{aligned}
$$

Hence

$$
t c P^{3}=Q^{2}+O\left(P^{4}, Q^{3}\right)
$$

where $c_{1}, c_{2}, c_{3}$ and $c$ are some positive constants.

At last we should think of the effect of the positive $r$-coordinates for the mapping $\psi$. From the expression (3.2), it is easily seen that $\frac{\partial R}{\partial r}>0$ for fixed $(p, r), p>0$. Therefore in the neighbourhood of the origin, $R(p, q, r)>$ $R(p, q, 0)$. Summing up the above results, we can figure out the location of $\psi(\{r>0\})$ as a domain lying above the back bone stratum. That is to say 
the $R$-coordinate $R_{0}$ of any point $\left(P_{0}, Q_{0}, R_{0}\right)$ of the domain $\psi(\{r>0\})$ is strictly larger than the coordinate $R_{1}$ of a point $\left(P_{0}, Q_{0}, R_{1}\right)$ located on the back bones. Hence $\psi(\{r>0\})$ cannot touch the back bones and required statement was shown.

\section{Applications}

As applications of the results in $\S 3$, we will look at two problems.

1. The estimate of error term in Weyl type formula.

2. The estimate of the eigenfunctions of Dirichlet problem to elliptic operators.

Among these, the second one is the direct motivation for the study of the oscillatory integrals in question.

\subsection{Weyl type formula}

Let us think of an operator $F(D)$ of order $M$ acting on the functions on torus $\mathbf{T}^{N}=(\mathbb{R} / 2 \pi \mathbb{Z})^{N}$.

$$
F(D) e^{i x \xi}=F(\xi) e^{i x \xi}
$$

The eigenvalues of this operator are evidently $\left\{F(z) ; z \in \mathbb{Z}^{N}\right\}$. Let us denote by $N(\lambda)$ the number of eigenvalues less than or equal to $\lambda^{M}$.

Then it is well known that

$$
N(\lambda)=\operatorname{vol}(D) \lambda^{N}+O\left(\lambda^{N-1}\right)
$$

where $D=\left\{\xi \in \mathbb{R}^{N} ; F(\xi) \leq 1\right\}$.

Our problem is to know more precise estimate of the asymptotic behaviour of $N(\lambda)$ as $\lambda \rightarrow \infty$ when

$$
F(\xi)=\xi_{1}^{4}+p \xi_{1}^{2} \xi_{2}^{2}+q \xi_{1} \xi_{2}^{3}+r \xi_{2}^{4} .
$$

In this respect Y. Colin de Verdière gave sufficiently general result.

Theorem 4.1 $[\mathrm{CV}] \quad$ Suppose $m$ is the maximum order of vanishing of the curvature of a curve $S=\partial D$ on $\mathbb{R}^{2}$.

If $m \geq 1$, the error estimate in (4.1) is $O\left(\lambda^{1-1 /(m+2)}\right)$.

Furthermore, if the normal direction to $S$ at the point where the curvature vanishes is rational, then this estimate is best possible.

Corollary 4.2 For the 4-th order operators with form (4.2), the error 
term in (4.1) can be estimated by

$$
\begin{aligned}
& O\left(\lambda^{3 / 4}\right) \text { for the cases }(3) \text { and }(5) \\
& O\left(\lambda^{2 / 3}\right) \quad \text { for the cases }(2) \text { and }(4) \text { of Theorem } 3.7 .
\end{aligned}
$$

Independent of Theorem 4.1, one can prove the following estimate of the error term by a simple way.

$$
\begin{array}{ll}
O\left(\lambda^{6 / 7}\right) & \text { for the cases (3) and (5) } \\
O\left(\lambda^{4 / 5}\right) & \text { for the cases (2) and (4) of Theorem 3.7. }
\end{array}
$$

The obtained result is the same one as in $[\mathrm{R}]$, but our proof is several times simpler than that by Randol.

Let us set $\chi_{\lambda}(\xi)=\chi(\xi / \lambda)$, where $\chi$ denotes the characteristic function of $D$. Then we have

$$
N(\lambda)=\sum_{z \in \mathbb{Z}^{2}} \chi_{\lambda}(z)
$$

Let $\rho(x)$ be a positive smooth function with support in the unit ball and $\int \rho(x) d x=1$.

We define the following mollified version of $N(\lambda)$

$$
N_{\varepsilon}(\lambda)=\sum_{z \in \mathbb{Z}^{2}}\left(\chi_{\lambda} * \rho_{\varepsilon}\right)(z)
$$

If $z$ lies a distance greater than $\varepsilon$ from the boundary of $\lambda D$, we see $\chi_{\lambda} *$ $\rho_{\varepsilon}(z)=\chi_{\lambda}(z)$.

So

$$
N_{\varepsilon}(\lambda-\varepsilon) \leq N(\lambda) \leq N_{\varepsilon}(\lambda+\varepsilon)
$$

If $N_{\varepsilon}(\lambda)$ satisfies the estimate

$$
N_{\varepsilon}(\lambda) \sim c \lambda^{2}+O\left(\lambda^{1+\alpha}\right)
$$

then

$$
c(\lambda-\varepsilon)^{2}+O\left(\lambda^{1+\alpha}\right) \leq N(\lambda) \leq c(\lambda+\varepsilon)^{2}+O\left(\lambda^{1+\alpha}\right)
$$

Let $\varepsilon=\lambda^{\alpha}$ then $(\lambda+\varepsilon)^{2}=\lambda^{2}+O\left(\lambda^{1+\alpha}\right)$. Therefore, we should effort to know the error estimate in (4.4). Applying Poisson's summation formula to 
(4.3) we get

$$
N_{\varepsilon}(\lambda)=\sum_{z \in \mathbb{Z}^{2}} \lambda^{2} \widehat{\chi}(2 \pi \lambda z) \widehat{\rho_{\varepsilon}}(2 \pi \lambda z)
$$

Now $\widehat{\chi}(0)=\operatorname{vol}(D)$ and $\widehat{\rho_{\varepsilon}}(0)=1$; the error term to be estimated is

$$
N_{\varepsilon}(\lambda)=\sum_{z \in \mathbb{Z}^{2} \backslash 0} \lambda^{2} \widehat{\chi}(2 \pi \lambda z) \widehat{\rho_{\varepsilon}}(2 \pi \lambda z) .
$$

We have

$$
\widehat{\chi}(\tau \omega)=\int_{D} e^{i \tau \omega \cdot \xi} d \xi=\frac{i}{\tau} \int_{\partial D} e^{i \tau \omega \cdot \xi} d S .
$$

This is nothing but the oscillatory integral (1.6) which has been studied in proving Theorem 3.7.

Thus we get

$$
\widehat{\chi}(\tau \omega)=O\left(\tau^{-3 / 2+\beta}\right)
$$

where $\beta=1 / 6$ (resp. 1/4) when the curvature vanishes for the first (resp. second) order. In view of (4.6) we can estimate (4.5) by

$$
\lambda^{2-3 / 2+\beta} \sum_{z \neq 0}|z|^{-3 / 2+\beta}\left(1+|\varepsilon z|^{2}\right)^{-N} \quad \text { with large } N
$$

or the integral

$$
\lambda^{1 / 2+\beta} \int \frac{1}{|x|^{3 / 2-\beta}}\left(1+|\varepsilon x|^{2}\right)^{-N} d x .
$$

Making the substitution $y=\varepsilon x$

$$
\lambda^{1 / 2+\beta} \varepsilon^{-1 / 2-\beta} \int \frac{1}{|y|^{3 / 2}}\left(1+|y|^{2}\right)^{-N} d y
$$

As this term shall be estimated by $(\lambda)^{1+\alpha}$ with $\varepsilon=\lambda^{\alpha}$, we have an equation with respect to $\alpha$ :

$$
\lambda^{1 / 2+\beta} \lambda^{\alpha(-1 / 2-\beta)}=\lambda^{1+\alpha} .
$$

So we get the aimed estimates

$$
\begin{aligned}
\alpha & =\frac{\beta-1 / 2}{\beta+3 / 2} \\
& =-\frac{1}{5} \quad \text { if the curvature vanishes for the first order }
\end{aligned}
$$




$$
=-\frac{1}{7} \text { if the curvature vanishes for the second order. }
$$

\subsection{The estimate of eigenfunctions of Dirichlet problem of ellip- tic operators}

In their paper [EK], Yu.V. Egorov and V.A. Kondrat'ev considered the following problem.

Let $F(D)$ be an elliptic PDE of order $2 m$ with constant coefficients:

$$
F(D)=\sum_{|\alpha|=2 m} a_{\alpha} D^{\alpha}
$$

Suppose we have a bounded domain $\Omega \subset \mathbb{R}^{N}$ with smooth boundary $\partial \Omega$. The eigenfunction $u_{\lambda}(x)$ of Dirichlet problem for the operator $F$ is considered:

$$
\begin{aligned}
F(D) u_{\lambda}(x) & =\lambda^{2 m} u_{\lambda} \quad x \in \Omega \\
u_{\lambda}(x) & =\frac{\partial}{\partial \nu} u_{\lambda}(x)=\cdots=\left(\frac{\partial}{\partial \nu}\right)^{2 m-1} u_{\lambda}(x)=0, \quad x \in \partial \Omega \\
\int_{\Omega}\left|u_{\lambda}(x)\right|^{2} d x & =1
\end{aligned}
$$

In [EK] the following estimate was given for the eigenfunction $u_{\lambda}$.

Theorem 4.3 Let $F(D)$ be such operator that $S=\left\{\xi \in \mathbb{R}^{N} ; F(\xi)-1=0\right\}$ has no point where the total curvature of $S$ vanishes. Then the eigenfunction $u_{\lambda}$ satisfies the following estimate:

$$
\left|u_{\lambda}(x)\right|^{2} \leq C|\lambda|^{N-1} \log |\lambda|
$$

for some constant $C>0$.

For these $F(D)$ that does not necessarily satisfy the condition imposed in Theorem 4.3, it is known that the estimate

$$
\left|u_{\lambda}(x)\right|^{2} \leq C|\lambda|^{N} .
$$

What we shall show is the following.

Theorem 4.4 For 4-th order elliptic operators of the form (4.2) the eigenfunction $u_{\lambda}$ of Dirichlet problem (4.7) satisfies the following estimate:

$$
\begin{aligned}
& \left|u_{\lambda}(x)\right|^{2} \leq C|\lambda|^{3 / 2} \quad \text { for the cases (3) and (5) } \\
& \left|u_{\lambda}(x)\right|^{2} \leq C|\lambda|^{4 / 3} \quad \text { for the cases (2) and (4) of Theorem 3.7. }
\end{aligned}
$$


Proof can be achieved almost in a parallel way to that of Theorem 4.3 except the point where the estimate on the fundamental solution $E(x)$ as in Theorem 3.7 shall be used in our case.

Let us suppose that $\max \left|u_{\lambda}\right|$ is attained at some point $x_{0} \in \Omega$ and $\operatorname{dist}\left(x_{0}, \partial \Omega\right)>c_{0}$ with some positive constant $c_{0}$. This can be guaranteed for $u_{\lambda}$ with $\lambda$ large enough as it is known from Lemma 4 of [EK] that the distance between the boundary $\partial \Omega$ and such point $x_{0}$ can be estimated from below by $d \lambda^{-1}$ for some positive constant $d$.

Set $y=\lambda x, v(y)=u_{\lambda}(x) \lambda^{-1 / 2}$, then $F\left(D_{y}\right) v(y)=v(y)$.

Let $h(y) \in \mathcal{C}_{0}^{\infty}\left(\mathbb{R}^{2}\right)$ such that $h(x)=0$ for $\left|y-y_{0}\right|>c_{0} \lambda$ where $y_{0}=\lambda x_{0}$ and $h(x)=1$ for $\left|y-y_{0}\right|<c_{0} \lambda / 2$. Then we have

$$
F\left(D_{y}\right) h(y) v(y)-h(y) v(y)=\sum_{|\alpha+\beta| \leq 2 m,|\alpha| \geq 1} k_{\alpha \beta} D^{\alpha} h(y) D^{\beta} v(y) .
$$

Let $E(x)$ be the fundamental solution of the operator $F-I$ studied in Theorem 3.7. The above expression becomes

$$
h(y) v(y)=\int E(y-z) \sum k_{\alpha \beta} D^{\alpha} h(z) D^{\beta} v(z) d z .
$$

Let us make a shift of coordinates that bring the origin to the point $y_{0}$. In this situation the integration above shall be done in the domain $c_{0} \lambda / 2<$ $|z|<c_{0} \lambda$. By the integration by parts we get

$$
h(0) v(0)=\int v(z) \sum k_{\alpha \beta}^{\prime} D^{\alpha} h(z) D^{\beta} E(-z) d z .
$$

Here we use the estimate of Theorem 3.6.

$$
\begin{aligned}
|v(0)|^{2}=|h(0) v(0)|^{2} & \leq\left. C \int|v(z)|^{2} \int \sum_{k_{\beta}}^{\prime} D^{\alpha} h(z) D^{\beta} E(-z)\right|^{2} d z . \\
& \leq C_{1} \lambda^{-2} \lambda^{-1+2 \beta} \int_{c_{0} \lambda / 2<|z|<c_{0} \lambda} d z \\
& \leq C_{2} \lambda^{-1+2 \beta} .
\end{aligned}
$$

where $\beta=1 / 6$ (resp. 1/4) for the cases (3) and (5) (resp. (2) and (4)) and $C_{2}$ is a positive constant not depending on $\lambda$. Thus we have gotten $\max \left|u_{\lambda}\right| \leq C_{2} \lambda^{1 / 2+\beta}$ and the required estimate was shown. 


\section{References}

[Ar] Arnol'd V.I., Wave front evolution and the equivalent Morse lemma. Comm. Pure Appl. Math. 29 (1976), 557-582.

[AVG] Arno'ld V.I., Varchencho A.N. and Gussein-Zade S.M., Singularities of Differentiable Maps, 1st part, Classification of critical points, caustics and wave fronts. Birkhäuser Verlag 1986.

[B1] Borovikov V.A., Fundamental solutions to linear partial differential equations. Trudy Moscovskogo Matematicheskogo Obschstva tom 8 (1959), (in Russian), 199-257.

[B2] Borovikov V.A., Some sufficient conditions for the absence of lacunas. Math. Sb. 55(98) (1961), (in Russian), 237-247.

[CV] Colin de Verdière Y.A., Nombre de points entiers dans une famille homothétique de domaines de $\mathbb{R}^{n}$. Ann. Scient. Ec. Norm. Sup. $4^{e}$ Série t. 10 (1977), 559-576.

[E] Erdélyi A., Asymptotic Expansions. Dover, 1956.

[EK] Egorov Yu.V. and Kondrat'ev V.A., On estimation of eigenfunctions of elliptic operators with constant coefficients. Trudy Seminara imeni I.M. Petrovskogo 12 (1987), (in Russian), 229-237.

[G] Gårding L., Sharp fronts of paired oscillatory integrals. Publ. RIMS, Kyoto Univ. 12 Suppl. (1977), 53-68.

[Gu] Guillemin V., Some classical Theorems in Spectral Theory Revisited. Seminar on Singularities of Solutions of LPDE. Annals of Math. Studies Vol. 91 (1981).

[H] Hörmander L., Fourier Integral Operators I. Acta Math. 127 (1971), 79-183.

[H-P] Hirschowitz A. and Piriou A., La propriété de transmission pour les distributions de Fourier; application aux lacunes. Séminaire Goulaouic-Schwartz 1976-1977, Exposé $n^{\circ} \mathrm{XIV}$.

[K] Kaneko A., Newton's diagram, Singular points, Oscillatory integrals. Lecture Notes in Mathematics Vol. 11, Sophia University (in Japanese), 1981.

[L] Leray J., Un prolongement de la transformation de Laplace qui transforme la solution unitaire d'un opérateur hyperbolique en sa solution élémentaire. (Problème de Cauchy IV), Bull. Soc. Math. France 90 (1962), 39-156.

[M] Malgrange B., Intégrales asymptotiques et monodromie. Ann. Scient. Ec. Norm. Sup. $4^{e}$ Série t. 7 (1974), 405-430.

[R] Randol B., A lattice point problem I. Trans. AMS 121 (1966), 257-268.

[Vai] Vainberg B.R., Asymptotic methods in Equations of Mathematical Physics. Moscow State University Publishers, 1982 (in Russian); English translation: Gordon and Breach Science Publishers, 1988.

[Var] Varchenko A.N., Newton polyhedra and Estimation of oscillating integrals. Functional Anal. Appl. Vol. 10 No.2 (1976), 175-196.

[Vas1] Vasiliev V.A., Sharpness and local Petrovski condition for hyperbolic operators with constant coefficients. Izvestia Seria Mat. Nauk SSSR, tom 50, No.2 (1986), (in Russian), 242-283.

[Vas2] Vasiliev V.A., Asymptotic exponential integrals, Newton diagram and the classi- 
fication of minimal points. Functional Anal. Appl. Vol 11 No.3 (1977), 1-11.

[Z] Zakalyukin V.M., Surgery on fronts and caustics depending on a parameter, versality of mappings. VINITI. Sovremennye Problemy Matematiki No.22 (1983), (in Russian), 59-93.

Institute of Control Sciences

Russian Academy of Sciences

Profsojunaja ul. 65, GSP-7

MOSCOW, 117806, Russia

E-mail: tanabe@ipu.rssi.ru, tanabe@mpim-bonn.mpg.de 